\title{
Higher Toda brackets and Massey products
}

\author{
Hans-Joachim Baues ${ }^{1}$. David Blanc ${ }^{2}$. \\ Shilpa Gondhali ${ }^{2}$
}

Received: 3 March 2015 / Accepted: 4 September 2015 / Published online: 26 November 2016

(C) Tbilisi Centre for Mathematical Sciences 2016

\begin{abstract}
We provide a uniform definition of higher order Toda brackets in a general setting, covering the known cases of long Toda brackets for topological spaces and Massey products for differential graded algebras, among others.
\end{abstract}

Keywords Higher order homotopy operation - Higher order cohomology operation · Toda bracket · Massey product · Chain complex · Enriched category · Path object . Monoidal model category

Mathematics Subject Classification Primary 18G55; Secondary 55S20 - 55S30 . $55 \mathrm{Q} 35 \cdot 18 \mathrm{D} 20$

\section{Introduction}

Toda brackets and Massey products have played an important role in homotopy theory ever since they were first defined in $[39,60,61]$ : in applications, such as $[2,5,23,35,55]$,

Dedicated to Ronnie Brown on the occasion of his eightieth birthday.

Communicated by Tim Porter and George Janelidze.

$凶$ Shilpa Gondhali

shilpa.s.gondhali@gmail.com; sgondhal@math.haifa.ac.il

Hans-Joachim Baues

baues@mpim-bonn.mpg.de

David Blanc

blanc@math.haifa.ac.il

1 Max-Planck-Institut für Mathematik, Vivatsgasse 7, 53111 Bonn, Germany

2 Department of Mathematics, University of Haifa, 31905 Haifa, Israel 


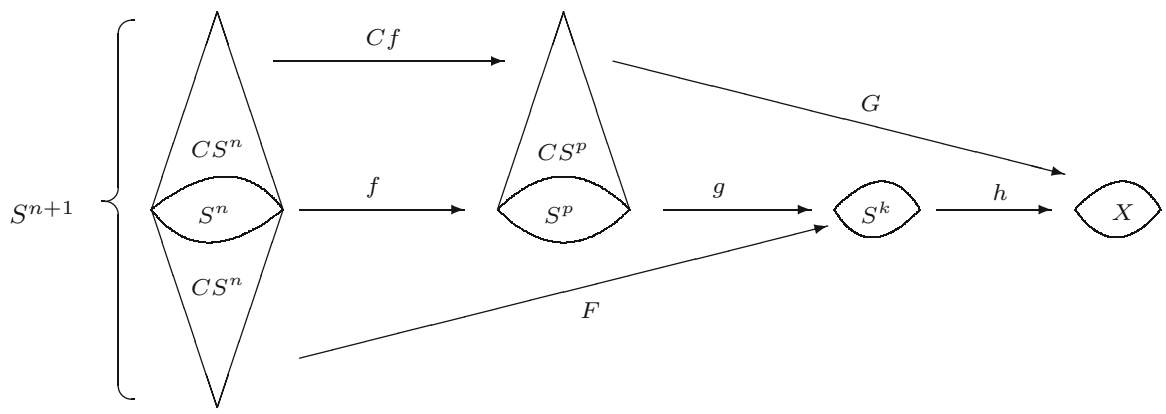

Fig. 1 The Toda bracket construction

and in a more theoretical vein, as in $[1,8,21,29,38,53,56]$. There are a number of variants (see, e.g., [3,20,42,47] and [6, Sect. 3.6.4]), as well as higher order versions including $[27,28,30,41,43,46,48,49,51,57,63]$. In recent years they have appeared in many other areas of mathematics, including symplectic geometry, representation theory, deformation theory, topological robotics, number theory, mathematical physics, and algebraic geometry (see [4,10,18, 19,26,31,32,52]).

Toda brackets were originally defined for diagrams of the form

$$
S^{n} \stackrel{f}{\rightarrow} S^{p} \stackrel{g}{\rightarrow} S^{k} \stackrel{h}{\rightarrow} X
$$

with $g \circ f$ and $h \circ g$ nullhomotopic.

If we choose nullhomotopies $F: g \circ f \sim 0$ and $G: h \circ g \sim 0$, they fit into a diagram of cones as in Fig. 1.

This yields an element $\langle h, g, f\rangle$ in $\left[S^{n+1}, X\right]$ called the Toda bracket. The value we get depends on the choices of nullhomotopies $F$ and $G$, so it is not uniquely determined. The Toda bracket is thus more properly a certain double coset of $h_{\# \pi_{n+1}}\left(S^{k}\right)+\Sigma f^{\#} \pi_{p+1}(X)$.

If we view $[h]$ as an element in $\pi_{*} X$, while $[g]$ is seen as a primary homotopy operation acting trivially on $[f]$ and $[h] \circ[g]=0$ is a relation among primary operations, we can think of the Toda bracket as a secondary homotopy operation. Similarly, a diagram of the form

$$
X \stackrel{f}{\rightarrow} K(G, n) \stackrel{g}{\rightarrow} K\left(G^{\prime}, p\right) \stackrel{h}{\rightarrow} K\left(G^{\prime \prime}, k\right)
$$

with $g \circ f \sim 0 \sim h \circ g$ defines a secondary cohomology operation in the sense of [2].

On the other hand, the Massey product in cohomology-defined whenever we have three classes $\alpha, \beta, \gamma \in H^{*} X$ with $\alpha \cdot \beta=0=\beta \cdot \gamma$-is a different type of secondary cohomology operation which does not fit into this paradigm.

All three examples have higher order versions, though the precise definitions are not always self-evident or unique (cf. [27,41,63]). Nevertheless, these higher order operations play an important role in homotopy theory-for instance, in enhancing our theoretical understanding of spectral sequences (cf. [9]) and in providing a conceptual full invariant for homotopy types of spaces (see $[13,59])$. 
The main goal of this note is to explain that higher order Toda brackets and higher Massey products have a uniform description, covering all cases known to the authors (including both the homotopy and cohomology versions).

The setting for our general notion of higher Toda brackets is any category $\mathcal{C}$ enriched in a suitable monoidal category $\mathcal{M}$. In fact, the minimal context in which higher Toda brackets can be defined is just an enrichment in a monoidal category equipped with a certain structure of "null cubes", encoded by the existence of an augmented path space functor $P X \rightarrow X$ satisfying certain properties (abstracted from those enjoyed by the usual path fibration of topological spaces). We call such an $\mathcal{M}$ a monoidal path category-see Sect. 2.

In this context we can define the notion of a higher order chain complex: that is, one in which the identity $\partial \partial=0$ holds only up to a sequence of coherent homotopies (see Sect. 3). This suffices to allow us to define the values of the corresponding higher order Toda bracket (see Sect. 4, where higher Massey products are also discussed).

However, in order for these Toda brackets to enjoy the expected properties, such as homotopy invariance, $\mathcal{M}$ must be also be a simplicial model category. In this case there is a model category structure on the category $\mathcal{M}$-Cat of categories enriched in $\mathcal{M}$, due to Lurie, Berger and Moerdijk, and others, in which the weak equivalences are Dwyer-Kan equivalences (see Definition 5.7). This is explained in Sect. 5, where we prove:

Theorem A Higher Toda brackets are preserved under Dwyer-Kan equivalences.

[See Theorem 5.14 below].

We also show that the usual higher Massey products in a differential graded algebra correspond to our definition (see Proposition 5.20).

In Sect. 6 we study the case of ordinary Toda brackets for chain complexes, and show their interpretation as secondary Ext-operations.

Notation 1.1 The category of sets will be denoted by Set, that of compactly generated topological spaces by Top (cf. [58], and compare [62]), and that of pointed compactly generated spaces by $\mathrm{Top}_{*}$.

If $R$ is a commutative ring with unit, the category of $R$-modules will be denoted by $\operatorname{Mod}_{R}$ (though that of abelian groups will be denoted simply by AbGp). The category of non-negatively graded $R$-modules will be denoted by $\operatorname{grMod}_{R}^{\geqslant 0}$, with objects $\mathcal{E}_{*}=\left\{E_{n}\right\}_{n \geq 0}$, and so on.

The category of $\mathbb{Z}$-graded chain complexes over $\operatorname{Mod}_{R}$ will be denoted by $\mathrm{Ch}_{R}$, with objects $\mathbf{A}_{*}, \mathbf{B}_{*}$, and so on, where

$$
\mathbf{A}_{*}:=\left(\cdots A_{n} \stackrel{\partial_{n}}{\longrightarrow} A_{n-1} \stackrel{\partial_{n-1}}{\longrightarrow} A_{n-2} \stackrel{\partial_{n-2}}{\longrightarrow} A_{n-3} \cdots\right) .
$$

The category of nonnegatively graded chain complexes over $\operatorname{Mod}_{R}$ will be denoted by $\mathrm{Ch}_{R}^{\geqslant 0}$. A chain map $f: \mathbf{A}_{*} \rightarrow \mathbf{B}_{*}$ inducing an isomorphism $f_{*}: H_{n} \mathbf{A}_{*} \longrightarrow H_{n} \mathbf{B}_{*}$ for all $n$ is called a quasi-isomorphism.

Finally, the category of simplicial sets will be denoted by $\mathcal{S}$, and that of pointed simplicial sets by $\mathcal{S}_{*}$. 


\section{Path functors in monoidal categories}

Higher order homotopy operations in a pointed model category $\mathcal{C}$, such as $\mathrm{Top}_{*}, \mathcal{S}_{*}$, or $\mathrm{Ch}_{R}$, are usually described in terms of higher order homotopies, which can be defined in turn in terms of an enrichment of $\mathcal{C}$ in an appropriate monoidal model category $\mathcal{M}$ (see, e.g., [12]). We here abstract the minimal properties of such an $\mathcal{M}$ needed for the construction of higher operations.

Definition 2.1 A monoidal path category is a functorially complete and cocomplete pointed monoidal category $\langle\mathcal{M}, \otimes, 1\rangle$, equipped with an path endofunctor $P: \mathcal{M} \rightarrow$ $\mathcal{M}$ and natural transformations $\mathfrak{p}_{X}: P X \rightarrow X, \theta^{L}: P X \otimes Y \rightarrow P(X \otimes Y)$, and $\theta^{R}: X \otimes P Y \rightarrow P(X \otimes Y)$.

We require that the following diagrams commute:

(a) Constant path combinations:
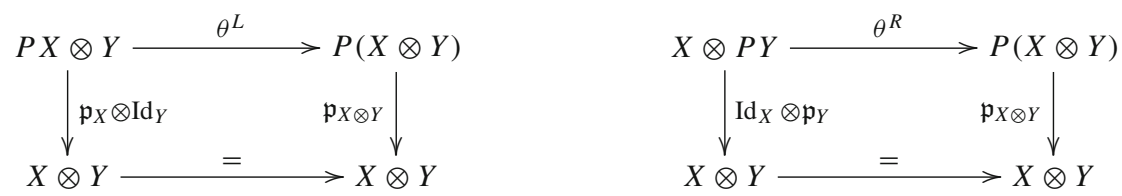

(b) Algebra structure (for each $k \geq 1$ ):



(c) Left and right constants:

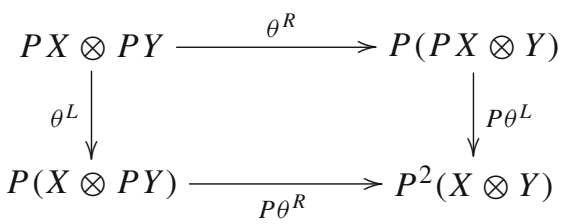

(d) From (2.3) we see that there are natural transformations

$$
\theta^{(i, j)}: P^{i} X \otimes P^{j} Y \rightarrow P^{i+j}(X \otimes Y)
$$

for any $i, j \geq 0$, defined

$$
\theta^{(i, j)}:=P^{i+j-1}\left(\theta^{L}\right) \circ \cdots \circ P^{j}\left(\theta^{L}\right) \circ P^{j-1}\left(\theta^{R}\right) \circ \cdots \circ \theta^{R} .
$$

These are required to be associative, in the obvious sense. 
(e) If we let $P^{n} X$ denote the result of applying the functor $P: \mathcal{M} \rightarrow \mathcal{M}$ to $X$ $n$ times (with $P^{0}:=\operatorname{Id}_{\mathcal{N}}$ ), we have $n+1$ different natural transformations $\partial_{i}^{n}: P^{n+1} X \rightarrow P^{n} X(i=0, \ldots, n)$, defined

$$
\partial_{i}=\partial_{i}^{n}:=P^{i}\left(\mathfrak{p}_{P^{n-i} X}\right)
$$

The natural transformations $\theta^{(i, j)}$ are required to satisfy the identities:

$$
\partial_{k}^{n-1} \circ \theta^{(i, j)}= \begin{cases}\theta^{(i-1, j)} \circ\left(\partial_{k}^{i-1} \otimes \mathrm{Id}\right) & \text { if } 0 \leq k<i \\ \theta^{(i, j-1)} \circ\left(\operatorname{Id} \otimes \partial_{k-i}^{j-1}\right) & \text { if } i \leq k<n\end{cases}
$$

for every $0 \leq k<i+j=n$.

Remark 2.2 The commutativity of (2.2) implies that the natural transformations of (2.4) satisfy the usual simplicial identities

$$
\partial_{i}^{n-1} \circ \partial_{j}^{n}=\partial_{j-1}^{n-1} \circ \partial_{i}^{n}
$$

for all $0 \leq i<j \leq n$ (see [64, Sect. 8.6]).

\subsection{Paths and cubes}

The natural setting where such path categories arise is when a monoidal category $\mathcal{M}$ is also simplicial, in the sense of [50, II, Sect. 1]. More specifically, we require the existence of an unpointed path functor $(-)^{I}: \mathcal{M} \rightarrow \mathcal{M}$ which behaves like a mapping space from the interval $[0,1]$, so we have natural transformations

(a) $e^{0}, e^{1}: X^{I} \rightarrow X$ (evaluation at the two endpoints),

(b) $s: X \rightarrow X^{I}$ with $e^{0} s=e^{1} s=$ Id (the constant path), and

(c) $\widetilde{\theta}^{L}: X^{I} \otimes Y \rightarrow(X \otimes Y)^{I}$ and $\widetilde{\theta}^{R}: X \otimes Y^{I} \rightarrow(X \otimes Y)^{I}$ (paths in a product).

These make the following diagrams commute:
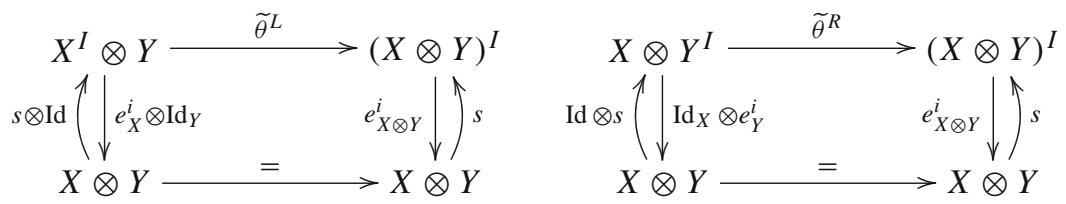
for $i=0,1$, as well as
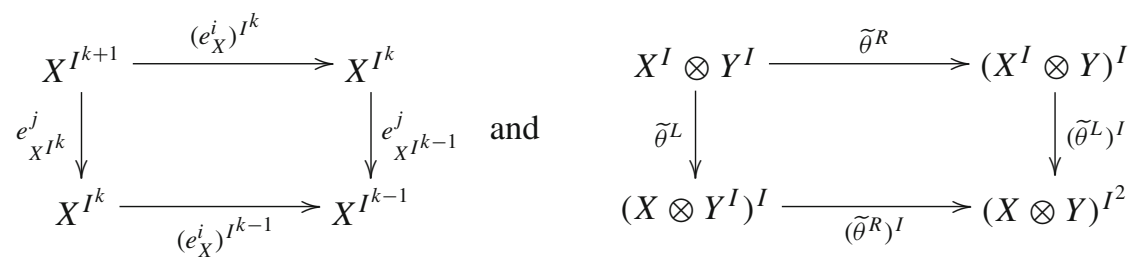

for all $k \geq 1$ and $i, j \in\{0,1\}$.

We may then define the required (pointed) path functor $P: \mathcal{M} \rightarrow \mathcal{M}$ by the functorial pullback diagram:

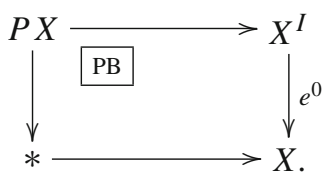

The commutativity of the right hand square in (2.8) allows us to define either composite to be the natural transformation $\widehat{\theta}^{(1,1)}: X^{I} \otimes Y^{I} \rightarrow(X \otimes Y)^{I^{2}}$.

We see that $\widetilde{\theta}^{L}$ induces a natural transformation $\theta^{L}: P X \otimes Y \rightarrow P(X \otimes Y)$, and similarly $\theta^{R}: X \otimes P Y \rightarrow P(X \otimes Y)$, making (2.1) commute.

Moreover, from (2.7) we see that (2.3) commutes, and that the natural transformations $\theta^{(i, j)}$ are associative and satisfy (2.5).

Example 2.3 The motivating example is provided by $\mathcal{M}=\mathrm{Top}_{*}$, with the monoidal structure given by the smash product $\otimes:=\wedge$, and $X^{I}:=\operatorname{map}_{*}(I, X)$ the mapping space out of the interval $I:=\Delta[1]_{+}$. Thus $P X$ is the usual pointed path space. Here $\operatorname{map}_{*}(X, Y)$ denotes the set $\operatorname{Hom}_{\operatorname{Top}_{*}}(X, Y)$ equipped with the compact-open topology.

Example 2.4 Similarly for $\mathcal{S}_{*}$, again with the smash product $\otimes:=\wedge$ and $X^{I}:=$ $\operatorname{map}_{*}\left(\Delta[1]_{+}, X\right)$, where $\operatorname{map}_{*}(X, Y) \in \mathcal{S}_{*}$ denotes the simplicial mapping space with $\operatorname{map}_{*}(X, Y)_{n}:=\operatorname{Hom}_{\mathcal{S}_{*}}\left(X \times \Delta[n]_{+}, Y\right)$.

When $X$ is a Kan complex, we can use Kan's model for $P X$, where $(P X)_{n}:=$ $\operatorname{Ker}\left(d_{1} d_{2} \ldots d_{n+1}: X_{n+1} \rightarrow X_{0}\right)$, and $\mathfrak{p}_{X}: P X \rightarrow X$ is $d_{0}^{i}$ in simplicial dimension $i$.

Example 2.5 Another variant is provided by a suitable category $\mathrm{Sp}$ of spectra with strictly associative smash product $\wedge$, such as the $S$-modules of [17], the symmetric spectra of [25], and the orthogonal spectra of [36]. One again has function spectra $\operatorname{map}_{S p}(X, Y)$, which can be used to define $X^{I}$ and $P X$. The unit is the sphere spectrum $S^{0}$.

Example 2.6 For chain complexes of $R$-modules we have a monoidal structure with the tensor product $\left(\mathbf{A}_{*} \otimes \mathbf{B}_{*}\right)_{n}:=\bigoplus_{i+j=n} A_{i} \otimes B_{j}$. 
Recall that the function complex $\underline{\operatorname{Hom}}\left(\mathbf{A}_{*}, \mathbf{B}_{*}\right)$ is given by

$$
\underline{\operatorname{Hom}}\left(\mathbf{A}_{*}, \mathbf{B}_{*}\right)_{n}:=\prod_{i \in \mathbb{Z}} \operatorname{Hom}\left(A_{i}, B_{i+n}\right),
$$

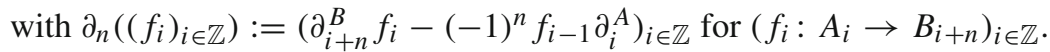

Thus for $\mathcal{M}=\mathrm{Ch}_{R}$ we may set $X^{I}:=\underline{\operatorname{Hom}}\left(C_{*}(\Delta[1] ; R), X\right)$, and see that $P \mathbf{A}_{*}$ has

$$
(P A)_{n}=A_{n} \oplus A_{n+1} \quad \text { with } \quad \partial\left(a, a^{\prime}\right)=\left(\partial a, \partial a^{\prime}+(-1)^{n+1} a\right),
$$

and $\mathfrak{p}_{\mathbf{A}_{*}}$ the projection.

\subsection{Cores and elements}

In any monoidal path category $\langle\mathcal{M}, \otimes, 1, P\rangle$ and for any $X \in \mathcal{M}$, we can think of $\operatorname{Hom}_{\mathcal{M}}(1, X)$ as the 'underlying set' of $X$, and think of a map $f: 1 \rightarrow X$ in $\mathcal{M}$ as an 'element' of $X$.

More generally, we may have a suitable monoidal subcategory $\mathcal{I}$ of $\mathcal{M}$, which we call a core, and define a generalized element of $X$ to be any map $f: \alpha \rightarrow X$ in $\mathcal{M}$ with $\alpha \in \mathcal{I}$.

Example 2.7 We may always choose $\mathcal{I}=\{1\}$ to consist of the unit of $\mathcal{M}$ alone. However, in some cases other natural choices are possible:

(a) In the three Examples of 2.3, 2.4, and 2.5, we can let $\mathcal{I}_{S}:=\left\{S^{n}\right\}_{n=0}^{\infty}$ consist of all (non-negative dimensional) spheres - this is evidently closed under $\otimes=\wedge$.

(b) In the category of chain complexes over a ring $R$ (Example 2.6), we let $\mathcal{I}_{R}:=$ $\left\{\widetilde{\mathbf{M}}(R, n)_{*}\right\}_{n \in \mathbb{Z}}$, where $\widetilde{\mathbf{M}}(R, n)_{*}$ is the Moore chain complex with $\widetilde{\mathbf{M}}(R, n)_{i}=$ $R$ for $i=n$, and 0 otherwise. Again we see that $\widetilde{\mathbf{M}}(R, p)_{*} \otimes \widetilde{\mathbf{M}}(R, q)_{*}=$ $\widetilde{\mathbf{M}}(R, p+q)_{*}$, so $\mathcal{I}_{R}$ is indeed a monoidal subcategory of $\left(\mathrm{Ch}_{R}, \otimes_{R}, \widetilde{\mathbf{M}}(R, 0)_{*}\right)$. We see that a generalized element in a chain complex $\mathbf{A}_{*}$ is now a map $f$ : $\widetilde{\mathbf{M}}(R, n)_{*} \rightarrow \mathbf{A}_{*}$ in $\mathrm{Ch}_{R}$-that is, an $n$-cycle in $\mathbf{A}_{*}$.

(c) Other examples are also possible-for example, if $\mathcal{I}^{\prime}:=\{\mathbf{M}(\mathbb{Z} / p, n)\}_{n=1}^{\infty}$ is the collection of $\bmod p$ Moore spaces, representing mod $p$ homotopy groups (see [45]), then it is not itself a monoidal subcategory of $\left(\operatorname{Top}_{*}, \wedge, S^{0}\right)$, since it is not closed under smash products. However, when $p$ is odd, the collection of finite wedges of such Moore spaces is monoidal, by [45, Corollary 6.6].

\section{Higher order chain complexes}

The structure defined in the previous section suffices to define higher order chain complexes, as in [9]: 


\subsection{Categories enriched in monoidal path categories}

Let $\mathcal{C}$ be a category enriched in a monoidal path category $\langle\mathcal{M}, \otimes, 1, P\rangle$, so that for any $a, b \in$ Obj $\mathcal{C}$ we have a mapping object $\operatorname{map}_{\mathcal{C}}(a, b)$ in $\mathcal{M}$, and for any $a, b, c \in$ Obj $\mathcal{C}$ we have a composition map

$$
\mu=\mu_{a, b, c}: \operatorname{map}_{\mathcal{C}}(b, c) \otimes \operatorname{map}_{\mathcal{C}}(a, b) \longrightarrow \operatorname{map}_{\mathcal{C}}(a, c)
$$

(written in the usual order for a composite), satisfying the standard associativity rules.

As in Sect. 2.2, we can think of a morphism $f: 1 \rightarrow \operatorname{map}_{\mathcal{C}}(a, b)$ in $\mathcal{M}$ as an 'element' of $\operatorname{map}_{\mathcal{C}}(a, b)$, or simply a map $f: a \rightarrow b$. In particular, we have 'identity $\operatorname{maps}^{\prime} \operatorname{Id}_{a}$ in $\operatorname{map}_{\mathcal{C}}(a, a)$ for each $a \in \operatorname{Obj} \mathcal{C}$, satisfying the usual unit rules.

In addition, a morphism $F: 1 \rightarrow P \operatorname{map}_{\mathcal{C}}(a, b)$ is called a nullhomotopy of $f:=\mathfrak{p}_{\operatorname{map}_{\mathrm{e}}(a, b)} \circ F$. Higher order nullhomotopies are defined by maps $F: 1 \rightarrow$ $P^{i} \operatorname{map}_{\mathcal{C}}(a, b)$.

The functoriality of $P$ implies that we can also compose (higher order) nullhomotopies by means of the composite of

$$
\begin{gathered}
P^{i} \operatorname{map}_{\mathcal{C}}(b, c) \otimes P^{j} \operatorname{map}_{\mathcal{C}}(a, b) \stackrel{\theta^{(i, j)}}{\longrightarrow} P^{i+j}\left[\operatorname{map}_{\mathcal{C}}(b, c) \otimes \operatorname{map}_{\mathcal{C}}(a, b)\right] \\
\stackrel{P^{i+j} \mu}{\longrightarrow} P^{i+j} \operatorname{map}_{\mathcal{C}}(a, c),
\end{gathered}
$$

which we denote by $\mu^{i, j}: P^{i} \operatorname{map}_{\mathcal{C}}(b, c) \otimes P^{j} \operatorname{map}_{\mathcal{C}}(a, b) \rightarrow P^{i+j} \operatorname{map}_{\mathcal{C}}(a, c)$. Again, the maps $\mu^{(-,-)}$are associative.

For a general core $\mathcal{I} \subseteq \mathcal{M}$ (cf. Sect. 2.2), we have generalized elements given by maps $f: \alpha \rightarrow \operatorname{map}_{\mathcal{C}}(a, b)$ for $\alpha \in \mathcal{I}$. We use the fact that $\mathcal{I}$ is a monoidal subcategory to define the composite of $f: \alpha \rightarrow \operatorname{map}_{\mathcal{C}}(a, b)$ with $g: \beta \rightarrow \operatorname{map}_{\mathcal{C}}(b, c)(\beta \in \mathcal{I})$ to be the composite in $\mathcal{M}$ of

$$
\beta \otimes \alpha \stackrel{g \otimes f}{\rightarrow} \operatorname{map}_{\mathcal{C}}(b, c) \otimes \operatorname{map}_{\mathcal{C}}(a, b) \stackrel{\mu}{\rightarrow} \operatorname{map}_{\mathcal{C}}(a, c),
$$

and similarly for generalized (higher order) nullhomotopies.

From (2.5) we see that:

$$
\partial_{k}^{n-1} \circ \mu^{i, j}= \begin{cases}\mu^{i-1, j} \circ\left(\partial_{k}^{i-1} \otimes \mathrm{Id}\right) & \text { if } 0 \leq k<i \\ \mu^{i, j-1} \circ\left(\operatorname{Id} \otimes \partial_{k-i}^{j-1}\right) & \text { if } i \leq k<i+j\end{cases}
$$

for every $0 \leq k<i+j=n$.

Remark 3.1 If the path structure $P$ comes from a unpointed path structure $(-)^{I}$ as in Sect. 2.1, a morphism $F: 1 \rightarrow \operatorname{map}_{\mathfrak{C}}(a, b)^{I}$ in $\mathcal{M}$ is called a homotopy $F: f_{0} \sim f_{1}$ between $f_{0}:=e_{\text {map }_{e}}^{0} \circ F$ and $f_{1}:=e_{\text {map }_{e}}^{1} \circ F$.

Higher order homotopies are defined by maps $F: 1 \rightarrow \operatorname{map}_{e} I^{I^{i}}(a, b)$, and the functoriality of $(-)^{I}$ implies that we can compose (higher order) homotopies by means of the composite of 


$$
\operatorname{map}_{\mathcal{C}}(b, c)^{I^{i}} \otimes \operatorname{map}_{\mathcal{C}}(a, b)^{I^{j}} \stackrel{\widehat{\theta}^{(i, j)}}{\longrightarrow}\left[\operatorname{map}_{\mathcal{C}}(b, c) \otimes \operatorname{map}_{\mathcal{C}}(a, b)\right]^{I^{i+j}} \stackrel{\mu^{I^{i+j}}}{\rightarrow} \operatorname{map}_{\mathcal{C}}(a, c)^{I^{i+j}}
$$

which we denote by $\widetilde{\mu}^{i, j}: \operatorname{map}_{\mathcal{C}}(b, c)^{I^{i}} \otimes \operatorname{map}_{\mathcal{C}}(a, b)^{I^{j}} \rightarrow\left(\operatorname{map}_{\mathcal{C}}(a, c)\right)^{I^{i+j}}$. These induce the maps $\mu^{i, j}$, as in Sect. 2.1.

Definition 3.2 Assume given a monoidal path category $\langle\mathcal{M}, \otimes, 1, P\rangle$ with core $\mathcal{I}$ in $\mathcal{M}$ (cf. Sect. 2.2), and choose an ordered set $\Gamma=\left(\gamma_{1}, \ldots, \gamma_{N}\right)$ of $N$ core elements.

An $n$-th order chain complex $\mathcal{K}=\left\langle K,\left\{\left\{F_{(i)}^{k}\right\}_{i=k+1}^{N}\right\}_{k=0}^{n}\right\rangle$ over $\mathcal{M}($ for $\Gamma$ ) of length $N \geq n+2$ consists of:

(a) A category $K$ enriched over $\mathcal{M}$, with $\operatorname{Obj}(K)=\left\{a_{0}, \ldots, a_{N}\right\}$ and

$$
\operatorname{map}_{K}\left(a_{i}, a_{j}\right)= \begin{cases}1 \amalg * & \text { if } i=j \\ * & \text { if } i<j\end{cases}
$$

$K$ will be called the underlying category of the $n$-th order chain complex $\mathcal{K}$.

(b) For each $0 \leq k \leq n$ and $i=k+1, \ldots N$, generalized elements

$$
F_{(i)}^{k}: \gamma_{i-k} \otimes \cdots \otimes \gamma_{i} \rightarrow P^{k} \operatorname{map}_{K}\left(a_{i}, a_{i-k-1}\right)
$$

such that

$$
\partial_{t} \circ F_{(i)}^{k}=\mu^{k-t-1, t}\left(F_{(i-t-1)}^{k-t-1} \otimes F_{(i)}^{t}\right)
$$

for all $0 \leq t<k$.

When $N=n+2$, we simply call $\mathcal{K}$ an $n$-th order chain complex.

Remark 3.3 Typically we are given a fixed category $\mathcal{C}$ enriched in a monoidal path category $\langle\mathcal{M}, \otimes, 1, P\rangle$, and the underlying category $K$ for a higher order chain complex $\mathcal{K}$ will simply be a finite subcategory of $\mathcal{C}$ (usually not full, because of condition (3.4)). Such a $\mathcal{K}$ will be called an $n$-th order chain complex in $\mathcal{C}$.

Definition 3.4 Given an $n$-th order chain complex $\mathcal{K}=\left\langle K,\left\{\left\{F_{(i)}^{k}\right\}_{i=k+1}^{N}\right\}_{k=0}^{n}\right\rangle$ over $\mathcal{M}$ (for $\Gamma$ ) of length $N$, and an enriched functor $\phi: K \rightarrow L$ over $\mathcal{M}$ (which we may assume to be the identity on objects, with $L$ also satisfying (3.4)), the induced $n$-th order chain complex $\mathcal{L}=\left\langle L,\left\{\left\{G_{(i)}^{k}\right\}_{i=k+1}^{N}\right\}_{k=0}^{n}\right\rangle$ over $\mathcal{M}$ (for the same $\Gamma$ ) is defined by setting

$$
G_{(i)}^{k}:=\phi\left(F_{(i)}^{k}\right): \gamma_{i-k} \otimes \cdots \otimes \gamma_{i} \rightarrow P^{k} \operatorname{map}_{L}\left(a_{i}, a_{i-k-1}\right)
$$

for all $0 \leq k \leq n$ and $k<i \leq N$

Remark 3.5 Note that we do not assume that we have $n$-th order nullhomotopies $F_{(i)}^{n}$ for each $i>n$ in $P^{n} \operatorname{map}_{K}\left(a_{i}, a_{i-n-1}\right)$ satisfying (3.5). 
However, from (3.5) and (3.3) we see that:

$$
\partial_{s} \circ \partial_{t} \circ F_{(i)}^{k}=\mu^{k-t-2, t}\left(\mu^{k-s-t-2, s}\left(F_{(i-s-t-2)}^{k-s-t-2} \otimes F_{(i-t-1)}^{s}\right) \otimes F_{(i)}^{t}\right)
$$

if $s+t<k-1$, and

$\partial_{s} \circ \partial_{t} \circ F_{(i)}^{k}=\mu^{k-t-1, t-1}\left(F_{(i-t-1)}^{k-t-1} \otimes \mu^{k-s-2, s+t-k+1}\left(F_{(i-s-t+k-2)}^{k-s-2} \otimes F_{(i)}^{s+t-k+1}\right)\right)$

if $k-1 \leq s+t$. Thus from the simplicial identity $\partial_{s} \circ \partial_{t}=\partial_{t-1} \circ \partial_{s}$ for $0 \leq s<t$ we deduce that the maps $\left\{F_{(i)}^{k}\right\}$ must satisfy:

$$
\mu\left(F_{(i-s-t-2)}^{r} \otimes F_{(i-t-1)}^{s} \otimes F_{(i)}^{t}\right)= \begin{cases}\mu\left(F_{(i-s-t-3)}^{r+1} \otimes F_{(i-s-1)}^{t-1} \otimes F_{(i)}^{s}\right) & \text { if } s<t \\ \mu\left(F_{(i-r-s-2)}^{t} \otimes F_{(i-r-1)}^{s} \otimes F_{(i)}^{r}\right) & \text { if } s \geq r \text { and } t=0 \\ \mu\left(F_{(i-r-t-3)}^{s+1} \otimes F_{(i-t-2)}^{r} \otimes F_{(i)}^{t-1}\right) & \text { if } s \geq r \text { and } t>0\end{cases}
$$

where we have simplified the notation using the associativity of $\mu$.

\subsection{A cubical description}

Higher order chain complexes were originally defined in [9, Sect. 4] in terms of a cubical enrichment, which is well suited to describing higher homotopies. In general, for an $(n-1)$-st order chain complex

$$
a_{n+1} \stackrel{F_{(n+1)}^{0}}{\longrightarrow} a_{n} \stackrel{F_{(n)}^{0}}{\longrightarrow} a_{n-2} \rightarrow \cdots \rightarrow a_{1} \stackrel{F_{(1)}^{0}}{\longrightarrow} a_{0},
$$

we may describe the choices of higher homotopies $F_{(i)}^{k}$ succinctly by arranging them as the collection of all the cubical faces in the boundary of $I^{n+2}$ containing a fixed vertex (which is indexed by $F_{(1)}^{0} \otimes F_{(2)}^{0} \otimes \cdots F_{(n)}^{0} \otimes F_{(n+1)}^{0}$ ).

The $k$-faces are indexed by

$$
F_{\left(i_{1}\right)}^{k_{1}} \otimes \cdots \otimes F_{\left(i_{r}\right)}^{k_{r}} \in P^{k_{1}} \operatorname{map}_{K}\left(a_{i_{1}}, a_{0}\right) \otimes \cdots \otimes P^{k_{r}} \operatorname{map}_{K}\left(a_{n+1}, a_{n-k_{r}}\right),
$$

with $\sum_{j=1}^{r} k_{j}=k, i_{j}=\sum_{t=1}^{j}\left(k_{t}+1\right)$, and $r=n-k+1$ (so $i_{1}=k_{1}+1$ and $\left.i_{r}=n+1\right)$.

By intersecting the corner of $\partial I^{n+2}$ with a transverse hyperplane in $\mathbb{R}^{n+1}$ we obtain an $(n+1)$-simplex $\sigma$, whose $n$-faces correspond to the $(n+1)$-facets of the corner, and so on. More precisely, the cone on this simplex (with cone point the chosen vertex $v$ of $\left.I^{n+2}\right)$ is homeomorphic to $I^{n+2}$, with each $(n+1)$-face of the cone obtained from an $(n+1)$-facet $\tau$ of the corner by identifying the $n$-corner opposite $v$ in $\tau$ to a single $n$-simplex in the base of the cone. See Fig. 2. 
Fig. 2 Corner of 3-cube and transverse 2 -simplex

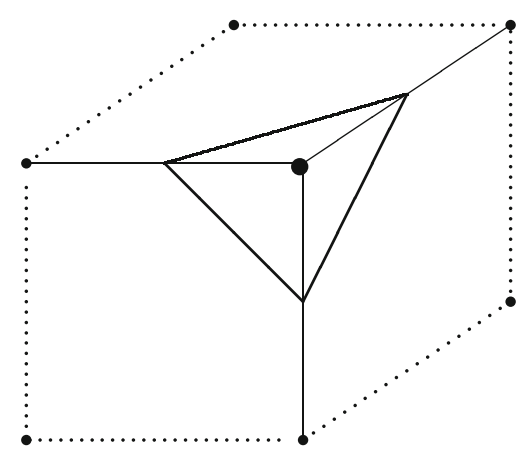

This explains why the maps $\partial_{i}^{n}: P^{n+1} X \rightarrow P^{n} X$ of Definition 2.1, which relate the various $\otimes$-composites appearing as facets of $\partial I^{n+1}$, satisfy simplicial, rather than cubical, identities.

Example 3.6 Consider a second order chain complex

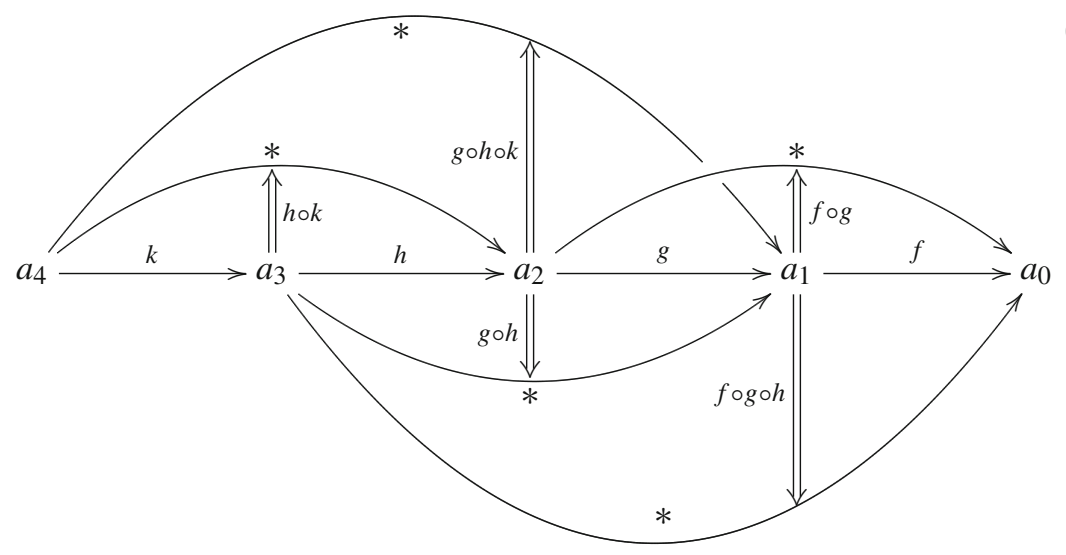

in Top Th , say, in which we have $n+1=4$ composable maps: $F_{(1)}^{0}=f, F_{(2)}^{0}=g$, and so on, with all adjacent composites nullhomotopic.

In this case we may choose nullhomotopies as indicated, namely: $F_{(2)}^{1}=f \circ g$ in $\operatorname{map}_{*}\left(a_{2}, a_{0}\right)^{I^{1}}$ (with $e^{0}(f \circ g)=*$ and $\left.e^{1}(f \circ g)=f g\right), F_{(3)}^{1}=g \circ h$ in $\operatorname{map}_{*}\left(a_{3}, a_{1}\right)^{I^{1}}$, and $F_{(4)}^{1}=h \circ k$ in $\operatorname{map}_{*}\left(a_{4}, a_{2}\right)^{I^{1}}$ - so that in fact $f \circ g$ is in the pointed path space $P \operatorname{map}_{*}\left(a_{2}, a_{0}\right)$. Similarly, $F_{(4)}^{2}=f \circ g \circ h$ is a homotopy of nullhomotopies between $h^{*}(f \circ g)$ and $f^{*}(g \circ h)$.

The more suggestive notation $f \circ g$, and so on, is motivated by the cubical BoardmanVogt $W$-construction of [14, Sect. 3], as explained in [9, Sect. 5]: we think a $k$-th order homotopy as a $k$-cube in the appropriate mapping spaces. 




Fig. 3 The cubical corner

If we apply the usual composition map

$$
\mu: \operatorname{map}_{*}\left(a_{2}, a_{1}\right) \otimes \operatorname{map}_{*}\left(a_{4}, a_{2}\right)^{I^{1}} \rightarrow \operatorname{map}_{*}\left(a_{4}, a_{1}\right)^{I^{1}}
$$

to $g \otimes h \circ k$, we obtain a nullhomotopy of $g h k$, and similarly for $g \circ h \otimes k$ in $\operatorname{map}_{*}\left(a_{3}, a_{1}\right)^{I^{1}} \otimes \operatorname{map}_{*}\left(a_{4}, a_{3}\right)$. Thus we may ask if these two nullhomotopies are themselves homotopic (relative to $g h k$ ): if so, we have a 2-cube $g \circ h \circ k$ in $\operatorname{map}_{*}\left(a_{4}, a_{1}\right)^{I^{2}}$, which in fact lies in $P^{2} \operatorname{map}_{*}\left(a_{4}, a_{1}\right)$. The "formal" post-composition with $f \in \operatorname{map}_{*}\left(a_{1}, a_{0}\right)$ yields $f \otimes g \circ h \circ k$ in $\operatorname{map}_{*}\left(a_{1}, a_{0}\right) \otimes P^{2} \operatorname{map}_{*}\left(a_{4}, a_{1}\right)$. Together with the other two formal composites $f \circ g \circ h \otimes k$ in $P^{2} \operatorname{map}_{*}\left(a_{3}, a_{0}\right) \otimes \operatorname{map}_{*}\left(a_{4}, a_{3}\right)$ and $f \circ g \otimes h \circ k$ in $P \operatorname{map}_{*}\left(a_{2}, a_{0}\right) \otimes P \operatorname{map}_{*}\left(a_{4}, a_{2}\right)$, it fits into the corner of the 3 -cube described in Fig. 3 (where we use both notations $F_{(2)}^{2}=f \circ g$, and so on, to label facets).

All vertices but the central one represent the zero map, and the dotted edges represent the trivial nullhomotopy of the zero map (and similarly for the invisible facets of the cube, representing the trivial second-order homotopy of the trivial nullhomotopy).

Remark 3.7 The cubical formalism may be used to describe the iterated path complex $P^{n} \mathbf{A}_{*}$ in the category of chain complexes (see Example 2.6):

We may use the conventions of Sect. 3.2 to identify the $k$-faces of the corner of an $n$-cube $I^{n}$ (adjacent to a fixed vertex $v$ ), for $0<k \leq n$, with the $(k-1)$-dimensional faces $\sigma_{(i)}^{k}$ of the standard $(n-1)$-simplex $\Delta[n-1]$ for $0 \leq i \leq\left(\begin{array}{l}n \\ k\end{array}\right)-1$ (see Fig. 2). Thus $I^{n}$ itself is labelled $\sigma_{(0)}^{n}$ (corresponding to $\Delta[n-1]$ ), with the $n(n-1)$-facets of $I^{n}$ adjacent to $v$ labelled $\sigma_{(0)}^{n-1}=d_{0} \sigma_{(0)}^{n}, \sigma_{(1)}^{n-1}=d_{1} \sigma_{(0)}^{n}$, and so on. The vertex $v$ is labelled $\sigma_{(0)}^{0}$ (not corresponding to any real face of $\left.\Delta[n-1]\right)$. 
Then

$$
\left(P^{n} A\right)_{j}=\bigoplus_{0 \leq k \leq n} \bigoplus_{0 \leq i<\left(\begin{array}{c}
n \\
k
\end{array}\right)} A_{j+k}^{\left[\sigma_{(i)}^{k}\right]}
$$

with the differential $\partial^{P^{n} A}:\left(P^{n} A\right)_{j} \rightarrow\left(P^{n} A\right)_{j-1}$ sending $a \in A_{j+k}^{\left[\sigma_{(i)}^{k}\right]}$ to $\partial^{A}(a)$ in the summand $A_{j+k-1}^{\left[\sigma_{(i)}^{k}\right]}$ of $\left(P^{n} A\right)_{j-1}$, and sending $a \in A_{j+k-1}^{\left[d_{t} \sigma_{(i)}^{k}\right]}$ to $(-1)^{n+k+t} a$ in the summand $A_{j+k-1}^{\left[\sigma_{(i)}^{k}\right]}$.

The structure maps $\partial_{i}^{n}: P^{n} \mathbf{A}_{*} \rightarrow P^{n-1} \mathbf{A}_{*}$ are given by the projections onto the summands labelled by the $i$-th simplicial facet of $\Delta[n]$ and its simplicial faces, for $0 \leq i \leq n-1$.

Example 3.8 The double path complex $P^{2} \mathbf{A}_{*}$ is given by

$$
\left(P^{2} A\right)_{j}=A_{j} \oplus A_{j+1} \oplus A_{j+1} \oplus A_{j+2},
$$

with

$$
\partial\left(a, b, b^{\prime}, c\right)=\left(\partial a, \partial b+(-1)^{j+1} a, \partial b^{\prime}+(-1)^{j+1} a, \partial c+(-1)^{j}\left(b-b^{\prime}\right)\right) .
$$

Example 3.9 Similarly, $\left(P^{3} A\right)_{j}$ is given by

$$
A_{j}^{\left[\sigma_{(0)}^{0}\right]} \oplus A_{j+1}^{\left[\sigma_{(0)}^{1}\right]} \oplus A_{j+1}^{\left[\sigma_{(1)}^{1}\right]} \oplus A_{j+1}^{\left[\sigma_{(2)}^{1}\right]} \oplus A_{j+2}^{\left[\sigma_{(0)}^{2}\right]} \oplus A_{j+2}^{\left[\sigma_{(1)}^{2}\right]} \oplus A_{j+2}^{\left[\sigma_{(2)}^{1}\right]} \oplus A_{j+3}^{\left[\sigma_{(0)}^{3}\right]}
$$

and

$$
\begin{aligned}
\partial\left(a, b_{0}, b_{1}, b_{2}, c_{0}, c_{1}, c_{2}, d\right)= & \left(\partial a, \partial b_{0}-\tau a, \partial b_{1}-\tau a, \partial b_{2}-\tau a,\right. \\
& \partial c_{0}+\tau\left(b_{1}-b_{0}\right), \partial c_{1}+\tau\left(b_{2}-b_{0}\right), \\
& \left.\partial c_{2}+\tau\left(b_{2}-b_{1}\right), \partial d-\tau\left(c_{2}-c_{1}+c_{1}\right)\right)
\end{aligned}
$$

for $\tau=(-1)^{j}$.

\section{Higher Toda brackets}

We now show how one may define the higher Toda bracket corresponding to a higher order chain complex. First, we need to define the object housing it:

Definition 4.1 In any monoidal path category $\langle\mathcal{M}, \otimes, 1, P\rangle$ we define the (modified) $n$-fold loop functor $\widetilde{\Omega}^{n}: \mathcal{M} \rightarrow \mathcal{M}$ to be the limit:

$$
\widetilde{\Omega}^{n} X:=\lim _{1 \leq k \leq n} P^{k} X
$$


where the limit is taken over all the natural maps $\partial_{i}^{k}: P^{k} X \rightarrow P^{k-1} X$ of Definition 2.1. By Sect. 3.2, we may think of this as a diagram indexed by the dual of the standard $n$-simplex.

The simplicial identities (2.6) imply that there is a natural map

$$
\widetilde{\sigma}_{X}^{n}: P^{n+1} X \rightarrow \widetilde{\Omega}^{n} X,
$$

which composes with the structure maps $\pi_{i}: \widetilde{\Omega}^{n} X \rightarrow P^{n} X$ for the limit to yield the face maps $\partial_{i}: P^{n+1} X \rightarrow P^{n} X(i=0, \ldots, n)$, since $\widetilde{\Omega}^{n} X$ is the $n$-th matching object for the restricted augmented simplicial object $P^{\bullet} X$ (cf. [22, Sect. 16.3.7]).

For $n=0$ we set $\widetilde{\Omega}^{0} X:=X$.

Example 4.2 By Sect. 3.2, we may think of (4.1) as the limit of a diagram indexed by the dual of the standard $n$-simplex. Thus $\widetilde{\Omega}^{1} X$ is the pullback in:

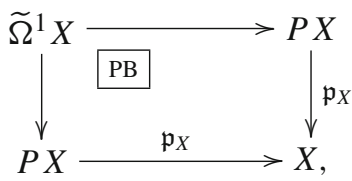

indexed by the inclusion of the two vertices into $\Delta[1]$, while $\widetilde{\Omega}^{2} X$ is the limit of the diagram:

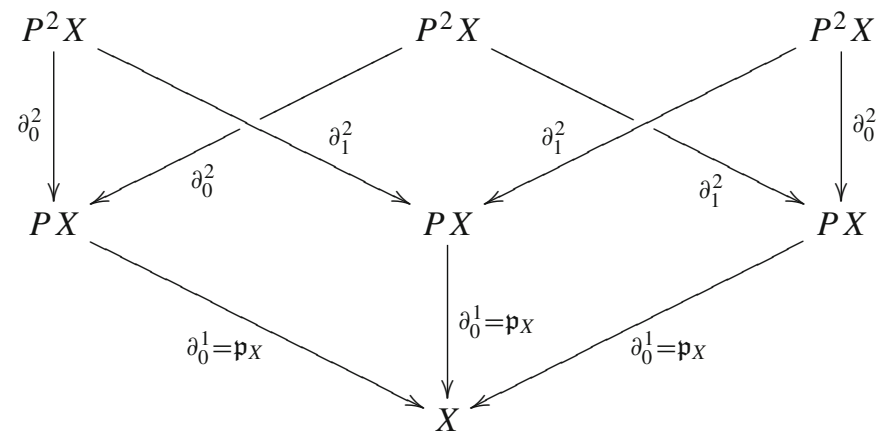

Definition 4.3 Let $\mathcal{K}$ be an $(n-1)$-st order chain complex (of length $n+1$ ) enriched in a monoidal path category $\langle\mathcal{M}, \otimes, 1, P\rangle$ (for a set $\Gamma=\left(\gamma_{1}, \ldots, \gamma_{n+1}\right)$ of core elements), as in Definition 3.2. If we apply the iterated composition map to each $k$-face of the form (3.8), we obtain an 'element'

$$
\mu\left(F_{\left(i_{1}\right)}^{k_{1}} \otimes \cdots \otimes F_{\left(i_{r}\right)}^{k_{r}}\right): \gamma_{1} \otimes \cdots \otimes \gamma_{n+1} \rightarrow P^{k} \operatorname{map}_{K}\left(a_{n+1}, a_{0}\right)
$$

(using the associativity of $\mu$ ),

From (3.5) and (3.3) we see that these elements (4.5) are compatible under the face maps $\partial_{t}: P^{k} \operatorname{map}_{K}\left(a_{n+1}, a_{0}\right) \rightarrow P^{k-1} \operatorname{map}_{K}\left(a_{n+1}, a_{0}\right)$, so that they fit together to 
define an element

$$
\langle\mathcal{K}\rangle: \gamma_{1} \otimes \cdots \otimes \gamma_{n+1} \rightarrow \widetilde{\Omega}^{n-1} \operatorname{map}_{K}\left(a_{n+1}, a_{0}\right)
$$

which we call the value of the $n$-th order Toda bracket associated to the chain complex $\mathcal{K}$.

If $\langle\mathcal{K}\rangle$ lifts along the map $\widetilde{\sigma}_{X}^{n-1}: P^{n} X \rightarrow \widetilde{\Omega}^{n-1} X$ of (4.2) (for $X:=$ $\left.\operatorname{map}_{K}\left(a_{n+1}, a_{0}\right)\right)$, we say that this value of the Toda bracket vanishes.

Remark 4.4 Given an $(n-1)$-st order chain complex $\mathcal{K}=\left\langle K,\left\{\left\{F_{(i)}^{k}\right\}_{i=k+1}^{n+1}\right\}_{k=0}^{n-1}\right\rangle$ over $\mathcal{M}$ (for $\Gamma$ ), any enriched functor $\phi: K \rightarrow L$ over $\mathcal{M}$ as in Definition 3.4 takes $\langle\mathcal{K}\rangle$ to

$$
\langle\mathcal{L}\rangle: \gamma_{1} \otimes \cdots \otimes \gamma_{n+1} \rightarrow \widetilde{\Omega}^{n-1} \operatorname{map}_{L}\left(a_{n+1}, a_{0}\right)
$$

where $\mathcal{L}$ is the $(n-1)$-st order chain complex induced by $\phi$, by functoriality of the limits in $\mathcal{M}$.

Example 4.5 Consider the second order chain complex $\mathcal{K}$ of (3.9), where for simplicity we may assume that $\gamma_{1}=\ldots \gamma_{3}=1$.

In $\mathrm{Top}_{*}$ the path functor is given by the usual simplicial structure, so $F_{(i)}^{k}$ (for $0 \leq k \leq 2$ and $i=k+1, \ldots, 4)$ can be thought of as a map from the $k$-cube $I^{k}$ to the mapping space $\operatorname{map}_{*}\left(a_{i}, a_{i-k-1}\right)$, sending the $k$-corner opposite the chosen vertex $v$ to the basepoint.

For $k=2$ we have three composites of the form (3.8), namely: $\mu\left(F_{(1)}^{0} \otimes F_{(4)}^{2}\right)$ (denoted by $f \otimes g \circ h \circ k$ in the notation of Example 3.6), $\mu\left(F_{(2)}^{1} \otimes F_{(4)}^{1}\right.$ ) (denoted by $f \circ g \otimes h \circ k$ ), and $\mu\left(F_{(3)}^{2} \otimes F_{(4)}^{0}\right)$ (denoted by $f \circ g \circ h \otimes k$ )—all in $P^{2} X$ for $X:=\operatorname{map}_{*}\left(a_{4}, a_{0}\right)$.

These were used in Fig. 3 to index the three 2-facets of the corner of $I^{2}$ adjacent to $v$ (which is itself indexed by the composite $\mu(f \otimes g \otimes h \otimes k)$ of the original maps in (3.9)).

As illustrated in Fig. 2, the two face maps $\partial_{0}^{2}, \partial_{1}^{2}: P^{2} X \rightarrow P^{1} X$ are shown in the cubical corner of Fig. 3 as the restrictions from the squares to the respective edges out of $v$ : thus $\partial_{0}^{2}\left(\mu\left(F_{(1)}^{0} \otimes F_{(4)}^{2}\right)\right)$ is denoted by $f \otimes g \otimes h \circ k$, while $\partial_{1}^{2}\left(\mu\left(F_{(1)}^{0} \otimes F_{(4)}^{2}\right)\right)$ is denoted by $f \otimes g \circ h \otimes k$, and so on.

As we see from Fig. 3, these simplicial face maps match up (as in Fig. 2) to yield an element in the limit of diagram (4.4), which is simply a map out of the 2-corner of the cube in Fig. 3 composed of the three visible two-facets, and sending the dotted boundary to the basepoint of $X$. The set of all such maps is precisely $\widetilde{\Omega}^{2} X$, and the value $\langle\mathcal{K}\rangle$ of the third order Toda bracket associated to $\mathcal{K}$ is the element of $\widetilde{\Omega}^{2} X$ we have just constructed.

\subsection{Massey products}

Massey products (and their higher order versions) also fit into our setting, although they cannot be defined as ordinary Toda brackets in a model category. This is because a (unital associative) differential graded algebra $\mathbf{A}_{*}$ over a commutative ground ring $R$ can 
be thought of as a category $\mathcal{C}$ with a single object $\xi$ enriched in $\left(\mathrm{Ch}_{R}, \otimes_{R}, \widetilde{\mathbf{M}}(R, 0)_{*}\right)$, with $\operatorname{Hom}_{\mathcal{C}}(\xi, \xi):=\mathbf{A}_{*}$.

In this context we choose the core of $\mathrm{Ch}_{R}$ to be $\mathcal{I}_{R}$ as in Example 2.7(b). Thus an $(n-1)$-st order chain complex in $\mathbf{A}_{*}$ consists of:

(a) The sequence of objects-necessarily $a_{i}=\xi$ for all $i$.

(b) A sequence of generalized maps $F_{(i)}^{0}: \tilde{\mathbf{M}}\left(R, m_{i}\right)_{*} \rightarrow \operatorname{Hom}_{\mathcal{C}}(\xi, \xi)$ for $i=$ $1, \ldots, n+1$, which may be identified with an $m_{i}$-cycle $H_{i}^{0} \in Z_{m_{i}} \mathbf{A}_{*}$ (see Example 2.7(b)).

(c) A sequence of generalized nullhomotopies $F_{(i)}^{1} \in P \mathbf{A}_{*}(i=2, \ldots, n+1)$, with $\mathfrak{p}_{\mathbf{A}_{*}}\left(F_{(i)}^{1}\right)=\mu\left(F_{(i-1)}^{0} \otimes F_{(i)}^{0}\right)$. From the description in Example 2.6 we see that $F_{(i)}^{1}$ is completely determined by an element $H_{i}^{1} \in A_{m_{i}+m_{i-1}+1}$ with $d\left(H_{i}^{1}\right)=H_{i-1}^{0} \cdot H_{i}^{0}$ (where $d$ is the differential and $\cdot$ is the multiplication in $\mathbf{A}_{*}$ ).

(d) From Example 3.8 we see that a 'second-order nullhomotopy' $F_{(i)}^{2} \in P^{2} \mathbf{A}_{*}$ $(i=3, \ldots, n+1)$, which is a $(j+2)$-cycle for $j:=m_{i}+m_{i-1}+m_{i-2}$, is determined uniquely by the element $H_{i}^{2} \in A_{j+2}$ (the last summand in (3.11)). From the last term in (3.12) we see that $F_{(i)}^{2}$ being a cycle means that

$$
d\left(H_{i}^{2}\right)=(-1)^{j+1}\left(H_{i-2}^{0} \cdot H_{i}^{1}-H_{i-1}^{1} \cdot H_{i}^{0}\right) .
$$

(e) In general, for each $1 \leq k<n$ and $i=k+1, \ldots n+1$, we have a (generalized) $F_{(i)}^{k} \in P^{k} \mathbf{A}_{*}$ which is a $(j+k)$-cycle for $j:=\sum_{t=i-k}^{i} m_{t}$, with

$$
\partial_{t} \circ F_{(i)}^{k}=F_{(i-t-1)}^{k-t-1} \cdot F_{(i)}^{t},
$$

and from the description in Remark 3.7 we see that again $F_{(i)}^{k}$ is completely determined by the component $H_{i}^{k}$ in the summand $A_{j+k}^{\left[\sigma_{(0)}^{k}\right]}$, with

$$
d\left(H_{i}^{k}\right)=(-1)^{k+j+1} \sum_{s=0}^{k-1}(-1)^{s} H_{i-k+s}^{s} \cdot H_{i}^{k-s-1} .
$$

Thus by Definition 4.3 we see that the value of the $n$-th order Toda bracket associated to this $(n-1)$-st order chain complex in $\mathbf{A}_{*}$ is the element in $\widetilde{\Omega}^{n-1} \mathbf{A}_{*}=$ $\lim _{1 \leq k<n} P^{k} \mathbf{A}_{*}$ determined by the coherent choice of elements

$$
H_{s+1}^{s} \cdot H_{n+1}^{n-1-s} \in A_{j+n-1} \quad \text { for } \quad s=0, \ldots, n-1,
$$

where $j:=\sum_{t=1}^{n} m_{t}$.

\section{Higher Toda brackets in model categories}

In order to define the values of higher Toda brackets, all we need is a category enriched in a monoidal path category $\mathcal{M}$. However, in applications we want to use such Toda 
brackets, either as obstructions to rectifying diagrams, or as invariants used in computations (e.g., of differentials in spectral sequence). For this we need to make an additional

Definition 5.1 A path model category is a pointed monoidal model category $\langle\mathcal{M}, \otimes, 1\rangle$ in the sense of $[24$, Ch. 4] which satisfies the conditions of either of [11, Theorems 1.9, 1.10], and which is also a simplicial model category as in [50, II, Sect. 2], equipped with a core $\mathcal{I}$ (cf. Sect. 2.2) consisting of cofibrant objects, and a natural transformation

$$
\zeta_{X, Y, K}: X^{K} \otimes Y^{K} \rightarrow(X \otimes Y)^{K}
$$

(natural in $X, Y \in \mathcal{M}$ and $K \in \mathcal{S}$ ).

Remark 5.2 By [24, Proposition 4.2.19], a path model category actually has a $\mathcal{S}_{*^{-}}$ model category structure-that is, we have functors $(-)^{K}: \mathcal{M} \rightarrow \mathcal{M}$ and $(-) \otimes K$ : $\mathcal{M} \rightarrow \mathcal{M}$ for every pointed simplicial set $K \in \mathcal{S}_{*}$, satisfying the usual axioms.

Examples 5.3 In practice we shall be interested only in the following examples:

(a) The monoidal structure on Top is cartesian, so we actually have a natural homeomorphism $\tilde{\zeta}: X^{K} \times Y^{K} \stackrel{\cong}{\rightrightarrows}(X \times Y)^{K}$. It is readily verified that in the pointed version $\left\langle\right.$ Top $\left._{*}, \wedge, S^{0}\right\rangle$ of Example 2.3, the map $\tilde{\zeta}$ induces $\zeta: X^{K} \wedge Y^{K} \rightarrow$ $(X \wedge Y)^{K}$.

(b) The monoidal structure on $\mathcal{S}$ is also cartesian, so in the pointed version $\left\langle\mathcal{S}_{*}, \wedge, S^{0}\right\rangle$ of Example 2.4 we also have an induced map as in (5.1).

(c) If we use symmetric spectra as our model for Sp (cf. Example 2.5) we see that the spectrum $X^{K}$ is defined levelwise, so we have (5.1) as for Top . .

(d) In the category $\left\langle\mathrm{Ch}_{R}, \otimes, \widetilde{\mathbf{M}}(R, 0)_{*}\right\rangle$ of chain complexes of $R$-modules (Example 2.6), the monoidal structure is not cartesian, but the simplicial structure is defined by setting $\mathbf{A}_{*}{ }^{K}:=\underline{\operatorname{Hom}}\left(C_{*} K, \mathbf{A}_{*}\right.$ ) (where $C_{*} K$ is the simplicial chain complex of $K \in \mathcal{S}$ ). The natural transformation (5.1) is induced by the diagonal $\Delta: K \rightarrow$ $K \times K$ in $\mathcal{S}$.

Note that all of these satisfy the hypotheses of one of [11, Theorems 1.9, 1.10], by [11, Sect. 1.8] and [33, Proposition A.3.2.4-A.3.2.24], so they are in fact path model categories.

Remark 5.4 In this case the simplicial structure defines the functor $(-)^{I}: \mathcal{M} \rightarrow \mathcal{M}$, with $X^{I}:=X^{\Delta[1]}$ (cf. [50, II, Sect. 1]), and $P X \hookrightarrow X^{\Delta[1]}$ is defined by the pullback (2.9). We can therefore identify $P^{k} X$ for each $k \geq 0$ with the subobject of $X^{[0,1]^{k}}$ consisting of all maps of the $k$-cube sending the corner opposite a fixed vertex to the basepoint (see Fig. 3).

Thus $\widetilde{\Omega}^{n} X$ is a subobject of $\lim _{k} \operatorname{map}_{*}\left([0,1]^{k}, X\right)$, which by adjunction may be identified with $X^{\operatorname{colim}_{k}[0,1]^{k}}$. Thus $\widetilde{\Omega}^{n} X$ itself is just $\operatorname{map}_{*}\left(\operatorname{colim} \widetilde{[0,1]^{k}}, X\right)$, where the colimit is now taken over all proper faces of $[0,1]^{n+1}$, and we identify the corner opposite our chosen vertex of $[0,1]^{n+1}$ to a point. This colimit is homeomorphic to 
an $n$-sphere, so $\widetilde{\Omega}^{n} X$ is homotopy equivalent to the $n$-fold loop space $\Omega^{n} X$, defined as usual by iterating the functor $\Omega: \mathcal{M} \rightarrow \mathcal{M}$ given by the pullback

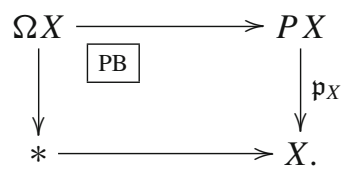

Remark 5.5 In any path model category $\mathcal{M}$, for any fibrant object $X$ we have an equivalence relation $\sim$ on the set of morphisms $\operatorname{Hom}_{\mathcal{M}}(1, X)$ (cf. Sect. 2.2), given by:

$$
f \sim g \Leftrightarrow \exists F: 1 \rightarrow X^{I} \quad \text { such that } e^{0} \circ F=f \quad \text { and } \quad e^{1} \circ F=g .
$$

We then define the (pointed) set of components $\pi_{0} X$ to be the set of equivalence classes in $\operatorname{Hom}_{\mathcal{N}}(1, X)$ under $\sim$.

Now let $\mathcal{C}$ be a category enriched in $\mathcal{M}$, and assume the mapping objects $\operatorname{map}_{\mathcal{C}}(a, b)$ are fibrant (e.g., if all objects in $\mathcal{M}$ are fibrant, as in $\operatorname{Top}_{*}$ ), If we denote $\pi_{0} \operatorname{map}_{\mathcal{C}}(a, b)$ simply by $[a, b]$, from Sect 3.1 we see that $\mu$ induces an associative composition on $[-,-]$, so that this serves as the set of morphisms in the homotopy category ho $\mathcal{C}$ of the $\mathcal{M}$-enriched category $\mathcal{C}$ (with the same objects as $\mathcal{C}$ ).

Definition 5.6 More generally, if $\mathcal{I}$ is the core of a path model category $\mathcal{M}$, for any core element $\gamma$ (which is cofibrant by Definition 5.1) the simplicial enrichment map $\operatorname{P}_{\mathcal{M}}$ in $\mathcal{M}$ allows us to identify $[\gamma, X]$ with $\pi_{0} \operatorname{map}_{\mathcal{M}}(\gamma, X)$ (see $[50$, II, 2.6]).

Thus if $\mathcal{C}$ is enriched in $\mathcal{M}$, we may set

$$
[a, b]_{\gamma}:=\pi_{0} \operatorname{map}_{\mathcal{M}}\left(\gamma, \operatorname{map}_{\mathcal{C}}(a, b)\right) .
$$

for any $a, b \in \mathcal{C}$ and $\gamma \in \mathcal{I}$.

Note that for any $\gamma, \delta \in \mathcal{I}$ and $i \geq 0$, the bifunctor $\otimes$, the map $\zeta_{X, Y, \Delta[i]}$ of (5.1) for $X:=\operatorname{map}_{\mathcal{C}}(b, c)$ and $Y:=\operatorname{map}_{\mathcal{C}}(a, b)$, and the composition $\mu: X \otimes Y \rightarrow Z$ (for $\left.Z:=\operatorname{map}_{\mathcal{C}}(a, c)\right)$ induce natural maps of sets

$$
\begin{aligned}
& \left(\operatorname{map}_{\mathcal{M}}(\gamma, X) \times \operatorname{map}_{\mathcal{M}}(\delta, Y)\right)_{i} \\
& \quad=\operatorname{Hom}_{\mathcal{M}}\left(\gamma, X^{\Delta[i]}\right) \times \operatorname{Hom}_{\mathcal{M}}\left(\delta, Y^{\Delta[i]}\right) \\
& \quad \stackrel{\otimes_{*}}{\longrightarrow} \operatorname{Hom}_{\mathcal{M}}\left(\gamma \otimes \delta, X^{\Delta[i]} \otimes Y^{\Delta[i]}\right) \stackrel{\zeta}{\rightarrow} \operatorname{Hom} \operatorname{Hi}_{\mathcal{M}}\left(\gamma \otimes \delta,(X \otimes Y)^{\Delta[i]}\right) \\
& \stackrel{\left(\mu^{\Delta[i]}\right)}{\longrightarrow} \operatorname{Hom}_{\mathcal{M}}\left(\gamma \otimes \delta, Z^{\Delta[i]}\right)=\left(\operatorname{map}_{\mathcal{M}}(\gamma \otimes \delta, Z)\right)_{i}
\end{aligned}
$$

and thus a composition map $v: \operatorname{map}_{\mathcal{M}}(\gamma, X) \times \operatorname{map}_{\mathcal{M}}(\delta, Y) \rightarrow \operatorname{map}_{\mathcal{M}}(\gamma \otimes \delta, Z)$ in $\mathcal{S}$. This induces an associative composition map

$$
\nu_{*}:[b, c]_{\gamma} \times[a, b]_{\delta} \rightarrow[a, c]_{\gamma \otimes \delta} .
$$


Thus we have an $\mathcal{I}$-graded category denoted by ho ${ }^{\mathcal{I}} \mathcal{C}$, called the $\mathcal{I}$-homotopy category of $\mathcal{C}$.

Definition 5.7 Assume given a path model category $\mathcal{M}$ with core $\mathcal{I}$. We say that a category $K$ enriched in $\mathcal{M}$ is fibrant if $\operatorname{map}_{K}(a, b)$ is fibrant in $\mathcal{M}$ for any $a, b \in K$. Note that since each $\gamma \in \mathcal{I}$ is cofibrant, this implies that $\operatorname{map}_{\mathcal{M}}\left(\gamma, \operatorname{map}_{K}(a, b)\right)$ is a fibrant simplicial set, by SM7.

An enriched functor $\phi: K \rightarrow L$ between categories $K$ and $L$ enriched in $\mathcal{M}$ is a Dwyer-Kan equivalence if

(a) For all $a, b \in \mathcal{C}, \phi: \operatorname{map}_{K}(a, b) \rightarrow \operatorname{map}_{L}(\phi(a), \phi(b))$ is a weak equivalence in $\mathcal{M}$.

(b) The induced functor $\phi_{*}:$ ho $^{\mathcal{I}} K \rightarrow$ ho $^{\mathcal{I}} L$ is an equivalence of $\mathcal{I}$-graded categories.

See [54], and compare [11].

We say that such a Dwyer-Kan equivalence is a trivial fibration if each $\phi$ : $\operatorname{map}_{K}(a, b) \rightarrow \operatorname{map}_{L}(\phi(a), \phi(b))$ is a fibration in $\mathcal{M}$.

By Definition 5.1 and [11, Theorems 1.9, 1.10] we have:

Theorem 5.8 There is a canonical model category structure on the category $\mathcal{M}$-Cat of small categories enriched in any path model category $\mathcal{M}$, in which the trivial fibrations and fibrant categories are defined object-wise, and the weak equivalences are the Dwyer-Kan equivalences.

Definition 5.9 Let $\mathcal{M}$ be a path model category with core $\mathcal{I}$, and let $\mathcal{K}^{(0)}=$ $\left.\left\langle K,\left\{F_{(i)}^{0}\right\}_{i=1}^{n+1}\right\}\right\rangle$ be a fixed fibrant 0 -th order chain complex of length $n+1$ over $\mathcal{M}$ for $\Gamma \subseteq \mathcal{I}$. We define $\mathcal{L}_{\mathcal{K}^{(0)}}$ to be the collection of all possible fibrant $(n-1)$-st order chain complexes $\mathcal{K}$ (of length $n+1$ ) extending $\mathcal{K}^{(0)}$.

Each $\mathcal{K} \in \mathcal{L}_{\mathcal{K}^{(0)}}$ has a value $\langle\mathcal{K}\rangle: \gamma_{1} \otimes \cdots \otimes \gamma_{n+1} \rightarrow \widetilde{\Omega}^{n-1} \operatorname{map}_{K}\left(a_{n+1}, a_{0}\right)$, as in (4.6), which we may identify with a 0 -simplex in the corresponding simplicial mapping space

$$
\langle\mathcal{K}\rangle \in \operatorname{map}_{\mathcal{M}}\left(\gamma_{1} \otimes \cdots \otimes \gamma_{n+1}, \widetilde{\Omega}^{n-1} \operatorname{map}_{K}\left(a_{n+1}, a_{0}\right)\right)_{0} .
$$

By Remark $5.4 \widetilde{\Omega}^{n-1} \operatorname{map}_{K}\left(a_{n+1}, a_{0}\right)$ is weakly equivalent to the $(n-1)$-fold loop space on the mapping $\operatorname{space}_{\operatorname{map}_{\mathcal{C}}}\left(a_{n+1}, a_{0}\right)$ in $\mathcal{M}$ (cf. [50, I, Sect. 2]). Moreover, we have a natural isomorphism

$$
\operatorname{map}_{\mathcal{M}}\left(Y, X^{L}\right) \stackrel{\simeq}{\rightarrow} \operatorname{map}_{\mathcal{S}}\left(L, \operatorname{map}_{\mathcal{M}}(Y, X)\right)
$$

for any $X, Y \in \mathcal{M}$ and $L \in \mathcal{S}$ any finite simplicial set, by [50, II, Sect. 1], so we may identify the path component $[\langle\mathcal{K}\rangle]$ of this 0 -simplex with the corresponding element in

$$
\begin{aligned}
\pi_{0} & \operatorname{map}_{\mathcal{M}}\left(\gamma_{1} \otimes \cdots \otimes \gamma_{n+1}, \Omega^{n-1} \operatorname{map}_{K}\left(a_{n+1}, a_{0}\right)\right) \\
& \cong \pi_{0} \Omega^{n-1} \operatorname{map}_{\mathcal{M}}\left(\gamma_{1} \otimes \cdots \otimes \gamma_{n+1}, \operatorname{map}_{K}\left(a_{n+1}, a_{0}\right)\right) \\
& \cong \pi_{n-1} \operatorname{map}_{\mathcal{M}}\left(\gamma_{1} \otimes \cdots \otimes \gamma_{n+1}, \operatorname{map}_{K}\left(a_{n+1}, a_{0}\right)\right)
\end{aligned}
$$


We call the set

$$
\left\langle\left\langle\mathcal{K}^{(0)}\right\rangle\right\rangle:=\left\{[\langle\mathcal{K}\rangle] \in \pi_{n-1} \operatorname{map}_{\mathcal{M}}\left(\gamma_{1} \otimes \cdots \otimes \gamma_{n+1}, \operatorname{map}_{K}\left(a_{n+1}, a_{0}\right)\right): \mathcal{K} \in \mathcal{L}_{\mathcal{K}^{(0)}}\right\}
$$

the $n$-th order Toda bracket for $\mathcal{K}^{(0)}$. We say that it vanishes if $0 \in\left\langle\left\langle\mathcal{K}^{(0)}\right\rangle\right\rangle$.

Of course, $\left\langle\left\langle\mathcal{K}^{(0)}\right\rangle\right\rangle$ may be empty (if there are no $(n-1)$-st order chain complexes $\mathcal{K}$ extending $\left.\mathcal{K}^{(0)}\right)$. It vanishes if and only if there is an $n$-th order chain complex extending $\mathcal{K}^{(0)}$.

Remark 5.10 When $K$ is a higher chain complex in $\mathcal{C}=\mathcal{M}$ in a monoidal path category enriched over itself (e.g., for $\mathcal{M}=\operatorname{Top}_{*}$ or $\mathcal{S}_{*}$ ), the homotopy class $[\langle K\rangle]$ may be thought of as an element in the group

$$
\left[\Sigma^{n-1} \gamma_{1} \otimes \cdots \otimes \gamma_{n+1} \otimes a_{n+1}, a_{0}\right]_{*}
$$

Moreover, $[\langle K\rangle]$ vanishes if and only if it represents the zero element in this group.

Lemma 5.11 In any simplicial model category $\mathcal{M}$ :

(a) Any (trivial) cofibration $i: K \hookrightarrow L$ in $\mathcal{S}$ induces a (trivial) fibration $i^{*}: X^{L} \rightarrow$ $X^{K}$, as long as $X \in \mathcal{M}$ is fibrant.

(b) Any (trivial) fibration $f: X \rightarrow Y$ in $\mathcal{M}$ induces a (trivial) fibration $f_{*}: X^{K} \rightarrow$ $Y^{K}$ for any (necessarily cofibrant) $K \in \mathcal{S}$.

Proof This follows from Axiom SM7 for $\mathcal{M}$, the natural isomorphism (5.5), and SM7 for $\mathcal{S}$ itself (cf. [50, II, Sects. 1-3]).

Lemma 5.12 If $\mathcal{M}$ is a simplicial model category and $f: X \rightarrow Y$ is a (trivial) fibration between fibrant objects in $\mathcal{M}$, then the induced maps $P^{k} f: P^{k} X \rightarrow P^{k} Y$ and $\widetilde{\Omega}^{k} f: \widetilde{\Omega}^{k} X \rightarrow \widetilde{\Omega}^{k} Y$ are (trivial) fibrations for all $k \geq 1$. Furthermore, if $f: X \rightarrow Y$ is a weak equivalence between fibrant and cofibrant objects in $\mathcal{M}$, so are $P^{k} f: P^{k} X \rightarrow P^{k} Y$ and $\widetilde{\Omega}^{k} f: \widetilde{\Omega}^{k} X \rightarrow \widetilde{\Omega}^{k} Y$.

Proof Let $C_{+}^{n}$ denote the sub-cubical set of the cube boundary $\partial I^{n}$ consisting of all facets adjacent to a fixed corner $v$ (i.e., the cubical star of $v$ in $\partial I^{n}$ ), with $\partial C^{n}$ its boundary (the cubical link of $v$ ), and similarly $C_{-}^{n}$ is the cubical star of the vertex $v^{\prime}$ diagonally opposite $v$ in $I^{n}$. The cofibration $i: \partial C_{+}^{n} \hookrightarrow C_{+}^{n}$ makes $i^{\#}: X^{C_{+}^{n}} \rightarrow X^{\partial C_{+}^{n}}$ a fibration in $\mathcal{M}$, by (a) of Lemma 5.11

In particular, the pullback square

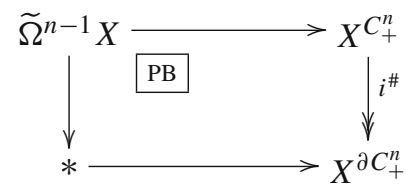

defining $\widetilde{\Omega}^{n-1} X$ (see Definition 4.1 and compare Sect. 3.2) is a homotopy pullback (see [40]). 
Thus if $f: X \rightarrow Y$ is a (trivial) fibration in $\mathcal{M}$, then the induced map $\widetilde{\Omega}^{n-1} f:$ $\widetilde{\Omega}^{n-1} X \rightarrow \widetilde{\Omega}^{n-1} Y$ is a (trivial) fibration, by (b) of Lemma 5.11

Similarly, if we consider the (pointed) cofibration sequence in $\mathcal{S}_{*}$ :

$$
S^{0}=\{0, *\} \hookrightarrow \Delta[1]_{+}=[0,1] \cup\{*\} \rightarrow \Delta[1]=[0,1]
$$

(with $*$ as basepoint in the first two, and 0 as the basepoint in the cofiber), we see from the corresponding fibration sequence in $\mathcal{N}$ :

$$
P X=X^{\Delta[1]} \hookrightarrow X^{I} \rightarrow X^{S^{0}}=X
$$

that if $f: X \rightarrow Y$ is a (trivial) fibration in $\mathcal{M}$, so is $P f: P X \rightarrow P Y$, by (b) of Lemma 5.11 again (see Remark 5.2 above).

Lemma 5.13 If $X$ is a fibrant object in a simplicial model category $\mathcal{M}$, then for each $n \geq 0$ the map $\widetilde{\sigma}_{X}^{n}: P^{n+1} X \rightarrow \widetilde{\Omega}^{n} X$ of (4.2) is a fibration.

Note that for $n=0, \widetilde{\Omega}^{0} X=X$ and $\widetilde{\sigma}_{X}^{0}$ is simply $\mathfrak{p}_{X}: P X \rightarrow X$.

Proof If we consider the map of cofibration sequences (pushouts to $*$ ) in $\mathcal{S}$ :

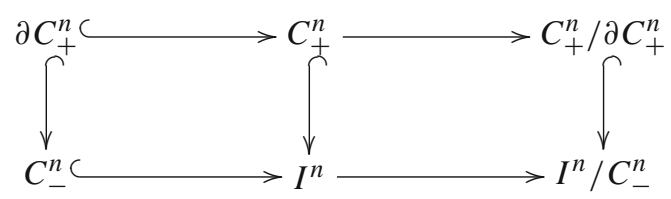

we see that the natural map $C_{+}^{n} / \partial C_{+}^{n} \rightarrow I^{n} / C_{-}^{n}$ is an inclusion (cofibration) in $\mathcal{S}_{*}$, so the natural map it induces-namely, $\widetilde{\sigma}_{X}^{n}: P^{n+1} X \rightarrow \widetilde{\Omega}^{n} X$ - is a fibration by (b) of Lemma 5.11.

For $n=0$ this follows directly because $\mathfrak{p}_{X}$ is a pullback in the following diagram:

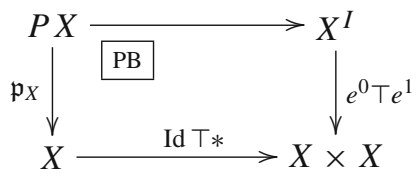

where $e^{0} \top e^{1}$ is a fibration since it is induced by the cofibration $\{0,1\} \hookrightarrow \Delta[1]$ in $\mathcal{S} . \square$

Theorem 5.14 Let $\mathcal{M}$ be a path model category with core $\mathcal{I}$, and let $\mathcal{K}^{(0)}=$ $\left.\left\langle K,\left\{F_{(i)}^{0}\right\}_{i=1}^{n+1}\right\}\right\rangle$ and $\left.\mathcal{L}^{(0)}=\left\langle L,\left\{G_{(i)}^{0}\right\}_{i=1}^{n+1}\right\}\right\rangle$ be 0 -th order chain complexes of length $n+1$ over $\mathcal{M}$ (for the same $\Gamma \subseteq \mathcal{I}$ ) with $K$ and $L$ fibrant, and let $\phi^{(0)}: \mathcal{K}^{(0)} \rightarrow \mathcal{L}^{(0)}$ be a map of 0 -th order chain complexes which is a Dwyer-Kan equivalence. Then the resulting equivalence of categories $\phi_{*}:$ ho $^{\mathcal{I}} K \rightarrow \mathrm{ho}^{\mathcal{I}}$ L induces a bijection between $\left\langle\left\langle\mathcal{K}^{(0)}\right\rangle\right\rangle$ and $\left\langle\left\langle\mathcal{L}^{(0)}\right\rangle\right\rangle$. 
Proof We assume for simplicity that $\phi$ is the identity on objects, so we may identify both $\pi_{0} \operatorname{map}_{\mathcal{M}}\left(\gamma, \operatorname{map}_{K}\left(a, a^{\prime}\right)\right)$ and $\pi_{0} \operatorname{map}_{\mathcal{M}}\left(\gamma, \operatorname{map}_{L}\left(a, a^{\prime}\right)\right)$ with $\left[a, a^{\prime}\right]_{\gamma}$. Similarly we may identify $\pi_{*} \operatorname{map}_{\mathcal{M}}\left(\gamma, \operatorname{map}_{K}\left(a, a^{\prime}\right)\right)$ and $\pi_{*} \operatorname{map}_{\mathcal{M}}\left(\gamma, \operatorname{map}_{L}\left(a, a^{\prime}\right)\right)$.

Given an $(n-1)$-st order chain complex $\mathcal{K}$ extending $\mathcal{K}^{(0)}, \phi$ induces an $(n-1)$ st order chain complex $\mathcal{L}$ extending $\mathcal{L}^{(0)}$, as in Definition 3.4, and takes the value $\langle\mathcal{K}\rangle \subset\left[a_{n+1}, a_{0}\right]_{\gamma_{1} \otimes \cdots \otimes \gamma_{n+1}}$ to $\langle\mathcal{L}\rangle$.

(a) First assume that $\phi^{(0)}: \mathcal{K}^{(0)} \rightarrow \mathcal{L}^{(0)}$ is a trivial fibration.

To show that the above correspondence is a bijection, let $\mathcal{L}$ be an $(n-1)$-st order chain complex extending $\mathcal{L}^{(0)}$. We show by induction on $k \geq 0$ that we have an $k$-th order chain complex $\mathcal{K}^{(k)}$ extending $\mathcal{K}^{(0)}$, where $\phi_{*} \mathcal{K}^{(k)}$ agrees with $\mathcal{L}$ to $k$-th order (by assumption this holds for $k=0$ ).

In the induction step, we have a $(k-1)$-st order chain complex $\mathcal{K}^{(k-1)}$ such that $\phi_{*} \mathcal{K}^{(k-1)}$ agrees with $\mathcal{L}$ to $(k-1)$-st order, which we wish to extend to $\mathcal{K}^{(k)}$. Thus we have a commuting diagram

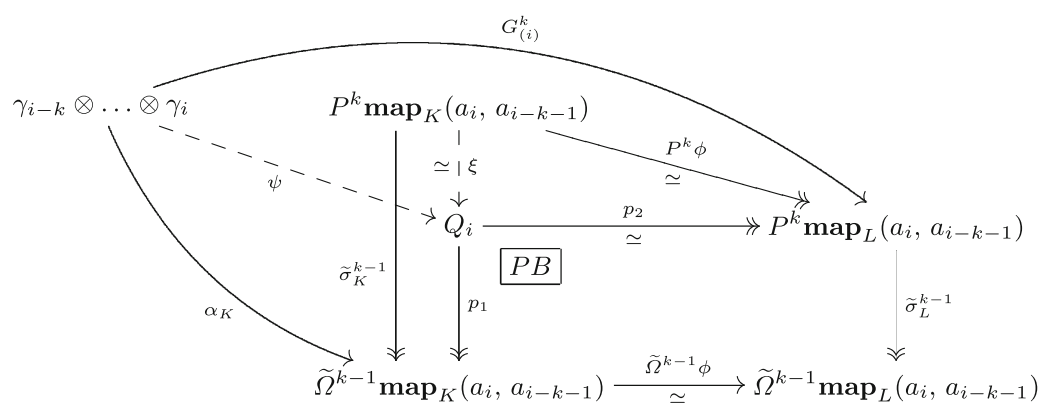

in which $Q_{i}$ is the pullback as indicated, and the map $\alpha_{K}: \gamma_{i-k} \otimes \cdots \otimes \gamma_{i} \rightarrow$ $\widetilde{\Omega}^{k-1} \operatorname{map}_{K}\left(a_{i}, a_{i-k-1}\right)$ is induced by the maps $F_{(t)}^{k-1}(t=0, \ldots, k-1)$, using (3.5) and (4.5).

Here $p_{2}$ is a trivial fibration and $p_{1}$ is a fibration by base change (using Lemmas 5.12 and 5.13). The maps $\psi: \gamma_{i-1} \otimes \gamma_{i} \rightarrow Q_{i}$ and $\xi: P^{k} \operatorname{map}_{K}\left(a_{i}, a_{i-k-1}\right) \rightarrow Q_{i}$ exist by the universal property, and $\xi$ is a weak equivalence by the 2 out of 3 property. Factor $\xi$ as

$$
P^{k} \operatorname{map}_{K}\left(a_{i}, a_{i-k-1}\right) \stackrel{j}{\rightarrow} P^{\widehat{k}^{\mathbf{m a p}}}{ }_{K}\left(a_{i}, a_{i-k-1}\right) \stackrel{\widehat{\xi}}{\rightarrow} Q_{i}
$$

where $j$ a trivial cofibration and $\widehat{\xi}$ is a trivial fibration. Since $\gamma_{i-k} \otimes \cdots \otimes \gamma_{i} \in \mathcal{I}$ is cofibrant, we have a lifting as indicated in the solid commuting square:

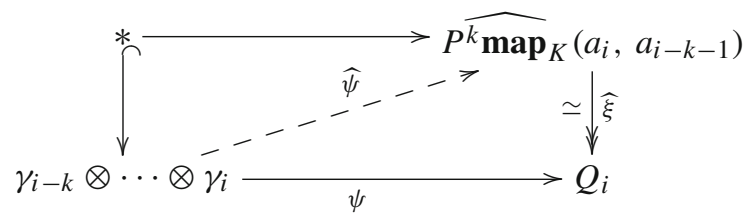


Since $j$ is a trivial cofibration and $\widetilde{\sigma}_{X}^{k}$ is a fibration (for $X:=\widetilde{\Omega}^{k-1} \operatorname{map}_{K}\left(a_{i}, a_{i-k-1}\right)$ ) by Lemma 5.13, we have a lift $\zeta$ as indicated in:

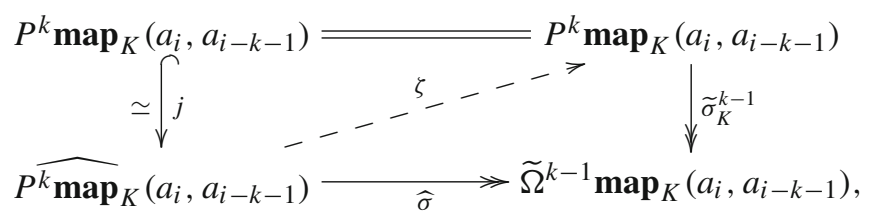

for $\widehat{\sigma}:=p_{1} \circ \widehat{\xi}$. Thus if we set $F_{(i)}^{k}: \gamma_{i-k} \otimes \cdots \otimes \gamma_{i} \rightarrow P^{k} \operatorname{map}_{K}\left(a_{i}, a_{i-k-1}\right)$ equal to $\zeta \circ \widehat{\psi}$, we see that

$$
\begin{aligned}
\partial_{t} \circ F_{(i)}^{k} & =\partial_{t} \circ \zeta \circ \widehat{\psi}=\pi_{t} \circ \widetilde{\sigma}_{K}^{k-1} \circ \zeta \circ \widehat{\psi}=\pi_{t} \circ p_{1} \circ \widehat{\xi} \circ \widehat{\psi} \\
& =\pi_{t} \circ p_{1} \circ \psi=\pi_{t} \circ \alpha_{K}=\mu^{k-t-1, t}\left(F_{(i-t-1)}^{k-t-1} \otimes F_{(i)}^{t}\right)
\end{aligned}
$$

(see Definition 4.1 and (3.5)) for all $0 \leq t<k$. Similarly,

$$
\begin{aligned}
P^{k} \phi \circ F_{(i)}^{k} & =P^{k} \phi \circ \zeta \circ \widehat{\psi}=p_{2} \circ \xi \circ \zeta \circ \widehat{\psi}=p_{2} \circ \widehat{\xi} \circ j \circ \zeta \circ \widehat{\psi} \\
& =p_{2} \circ \widehat{\xi} \circ \widehat{\psi}=G_{(i)}^{k} .
\end{aligned}
$$

Thus by induction we see that any $(n-1)$-st order chain complex $\mathcal{L}^{(n-1)}$ extending $\mathcal{L}^{(0)}$ lifts along $\phi$ to $\mathcal{K}^{(n-1)}$, so that $\phi_{*}$ is surjective.

On the other hand, since $\phi$ is a trivial fibration in map $\operatorname{\mathcal {N}}_{\mathcal{N}}$, in particular $\widetilde{\Omega}^{n-1} \phi: \widetilde{\Omega}^{n-1} \operatorname{map}_{K}\left(a_{n+1}, a_{0}\right) \rightarrow \widetilde{\Omega}^{n-1} \operatorname{map}_{L}\left(a_{n+1}, a_{0}\right)$ is a trivial fibration in $\mathcal{M}$, so it induces an isomorphism

$\pi_{n-1} \operatorname{map}_{\mathcal{M}}\left(\gamma_{1} \ldots \gamma_{n+1}, \operatorname{map}_{K}\left(a_{n+1}, a_{0}\right)\right) \stackrel{\cong}{\rightrightarrows} \pi_{n-1} \operatorname{map}_{\mathcal{M}}\left(\gamma_{1} \ldots \gamma_{n+1}, \operatorname{map}_{L}\left(a_{n+1}, a_{0}\right)\right)$

by SM7.

Thus if $\mathcal{K}^{\prime}$ is some other extension of $\mathcal{K}^{(0)}$ to an $(n-1)$-st order chain complex, and $\left[\left\langle\phi_{*} \mathcal{K}\right\rangle\right]=\left[\left\langle\phi_{*} \mathcal{K}^{\prime}\right\rangle\right]$ in $\pi_{n-1} \operatorname{map}_{\mathcal{M}}\left(\gamma_{1} \ldots \gamma_{n+1}, \operatorname{map}_{L}\left(a_{n+1}, a_{0}\right)\right)$, then $[\langle\mathcal{K}\rangle]=$ $\left[\left\langle\mathcal{K}^{\prime}\right\rangle\right]$ in $\pi_{n-1} \operatorname{map}_{\mathcal{M}}\left(\gamma_{1} \ldots \gamma_{n+1}, \operatorname{map}_{K}\left(a_{n+1}, a_{0}\right)\right)$.

We can see directly that $\left\langle\mathcal{L}^{(n-1)}\right\rangle$ vanishes if and only if it lifts to $F_{(n+1)}^{n}: \gamma_{0} \otimes$ $\cdots \otimes \gamma_{n+1} \rightarrow P^{n+1} \operatorname{map}_{K}\left(a_{n+1}, a_{0}\right)$, this happens if and only if the corresponding value $\left\langle\mathcal{K}^{(n-1)}\right\rangle$ vanishes, too.

(b) Now assume that $\phi^{(0)}: \mathcal{K}^{(0)} \rightarrow \mathcal{L}^{(0)}$ is an arbitrary weak equivalence, but that $\mathcal{K}^{(0)}$ and $\mathcal{L}^{(0)}$ are both fibrant and cofibrant. Factoring $\phi^{(0)}$ as a trivial cofibration followed by a trivial fibration, by (a) it suffices to assume that $\phi^{(0)}$ is a trivial cofibration. This implies that we have a lifting as indicated in the diagram of $\mathcal{M}$-categories 


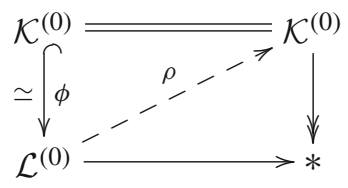

using Theorem 5.8. Thus by [24, Proposition 1.2.8], $\phi$ is a homotopy equivalence (with strict left inverse $\rho$ ).

Assume now that $\left(\mathcal{L}^{(0)}\right)^{\mathcal{J}}$ is a path object for $\mathcal{L}^{(0)}$ in $\mathcal{M}$-Cat (cf. [50, I, Sect. 1]), equipped with two trivial fibrations $d_{0}, d_{1}:\left(\mathcal{L}^{(0)}\right)^{\mathcal{J}} \rightarrow \mathcal{L}^{(0)}$-for example, we may apply the unpointed path functor $(-)^{I}$ of Sect. 2.1 objectwise to the mapping spaces of $\mathcal{L}^{(0)}$. A right homotopy $\phi \circ \rho \sim$ Id is then given by $H: \mathcal{L}^{(0)} \rightarrow\left(\mathcal{L}^{(0)}\right)^{\mathcal{J}}$, and $d_{0}, d_{1}$ induce the required bijection by (a).

(c) Finally, if $\phi: \mathcal{K}^{(0)} \rightarrow \mathcal{L}^{(0)}$ is any Dwyer-Kan equivalence, with cofibrant replacements $\psi: \widehat{\mathcal{K}}^{(0)} \rightarrow \mathcal{K}^{(0)}$ and $\xi: \widehat{\mathcal{L}}^{(0)} \rightarrow \mathcal{L}^{(0)}$ in $\mathcal{M}$-Cat (so both $\psi$ and $\xi$ are trivial fibrations), we have a lifting

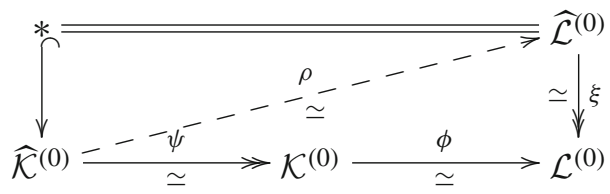

where $\rho$ is a Dwyer-Kan equivalence between fibrant and cofibrant $\mathcal{M}$-categories, so it induces a bijection as required by (b), while $\psi$ and $\xi$ are trivial fibrations in $\mathcal{M}$-Cat, so they induce the required bijections by (a). Since the lower right quadrangle in (5.12) commutes, $\phi$ also induces a bijection as required.

Definition 5.15 Given a path model category $\mathcal{M}$ with core $\mathcal{I}$, let $\mathcal{C}$ be a (small) subcategory of $\mathcal{M}$-Cat consisting of fibrant 0 -th order chain complexes of length $N=n+1$ for $\Gamma \subseteq \mathcal{I}$. If $\sim$ is the equivalence relation on $\mathcal{C}$ generated by Dwyer-Kan equivalences, let $\mathrm{Ho}^{\Gamma} \mathrm{C}:=\mathrm{e} / \sim$. An equivalence class in $\mathrm{Ho}^{\Gamma} \mathcal{C}$ will be called a homotopy chain complex for $\Gamma$.

Example 5.16 Our motivating example is when $\mathcal{C}$ is an $\mathcal{M}$-subcategory of a model category $\mathrm{C}^{\prime}$, whose weak equivalences $f: X \rightarrow Y$ between fibrant objects are maps inducing an isomorphism $f_{*}: \pi_{*} \operatorname{map}_{\mathcal{M}}\left(\gamma, \operatorname{map}_{\mathcal{C}^{\prime}}(Z, X)\right) \rightarrow \pi_{*} \operatorname{map}_{\mathcal{M}}\left(\gamma, \operatorname{map}_{\mathcal{C}^{\prime}}(Z, Y)\right)$ for every cofibrant $Z \in \mathcal{C}^{\prime}$ and every $\gamma \in \mathcal{I}$. Examples include those of 2.3-2.6 with $\mathcal{I}$ as in Example 2.7.

In this case a homotopy chain complex $\Lambda$ of length $n+1$ in $\mathrm{Ho}^{\Gamma} \mathcal{C}$ is represented by a sequence of elements

$$
\varphi_{i} \in\left[a_{i}, a_{i-1}\right]_{\gamma_{i}} \cong \pi_{0} \operatorname{map}_{\mathcal{M}}\left(\gamma_{i}, \operatorname{map}_{\mathcal{C}}\left(a_{i}, a_{i-1}\right)\right) \quad(i=1, \ldots, n+1)
$$

such that

$$
\mathcal{v}_{*}\left(\varphi_{i-1}, \varphi_{i}\right)=0 \quad \text { in }\left[a_{i}, a_{i-2}\right]_{\gamma_{i-1} \otimes \gamma_{i}} \quad(i=2, \ldots n+1),
$$

in the notation of Definition 5.6. 
In particular, when $\mathcal{I}=\{1\}, \Lambda$ may be described by a diagram:

$$
a_{n+1} \stackrel{\varphi_{n+1}}{\longrightarrow} a_{n} \stackrel{\varphi_{n}}{\rightarrow} a_{n-2} \rightarrow \cdots \rightarrow a_{1} \stackrel{\varphi_{1}}{\rightarrow} a_{0},
$$

in ho $\mathrm{C}^{\prime}$ such that $\varphi_{i-1} \circ \varphi_{i}=0$ for $i=2, \ldots n+1$.

However, in the context of Massey products (cf. Sect. 4.1), we do not have such a model category $\mathrm{C}^{\prime}$ available. In this case, we let $\mathcal{C}$ be a set of DGAs over $R$ with a given homology algebra, $\Gamma=\mathcal{I}_{R}$ as in Example 2.7(b), and a homotopy chain complex $\Lambda$ in $\mathrm{Ho}^{\Gamma} \mathrm{C}$ is a quasi-isomorphism class of DGAs in $\mathrm{C}$.

Definition 5.17 Given a path model category $\mathcal{M}$ with core $\mathcal{I}$, a category $\mathcal{C}$ as in Definition 5.15 for $\Gamma \subseteq \mathcal{I}$, and a homotopy chain complex $\Lambda$ of length $n+1$ for $\Gamma$, the corresponding $n$-th order Toda bracket $\langle\langle\Lambda\rangle\rangle$ is defined to be $\left\langle\left\langle\mathcal{K}^{(0)}\right\rangle\right\rangle \subseteq$ $\pi_{n-1} \operatorname{map}_{\mathcal{M}}\left(\gamma_{1} \otimes \cdots \otimes \gamma_{n+1}, \operatorname{map}_{K}\left(a_{n+1}, a_{0}\right)\right)$ for some representative $\mathcal{K}^{(0)}$ of $\Lambda$.

By Theorem 5.14 we see:

Lemma 5.18 The $n$-th order Toda bracket $\langle\langle\Lambda\rangle\rangle$ is well-defined.

Remark 5.19 As usual, for a homotopy chain complex $\Lambda$ :

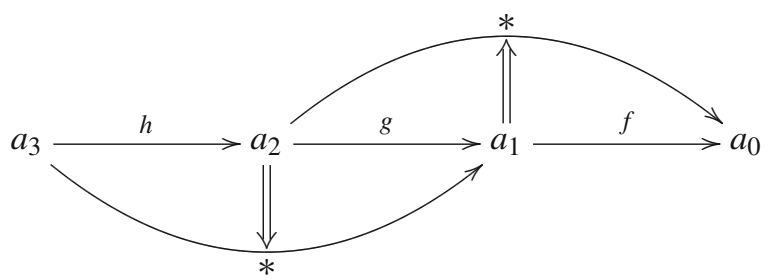

of length 3 , we can identify the corresponding secondary Toda bracket

$$
\begin{aligned}
\langle\langle\Lambda\rangle\rangle & \subseteq \pi_{1} \operatorname{map}_{\mathcal{M}}\left(\gamma_{1} \otimes \gamma_{2} \otimes \gamma_{3}, \operatorname{map}_{K}\left(a_{3}, a_{0}\right)\right) \\
& \cong \pi_{0} \operatorname{map}_{\mathcal{M}}\left(\gamma_{1} \otimes \gamma_{2} \otimes \gamma_{3}, \operatorname{man}_{K}\left(a_{3}, a_{0}\right)\right)
\end{aligned}
$$

as a double coset of the subgroups $h^{*} \pi_{0} \operatorname{map}_{\mathcal{M}}\left(\gamma_{1} \otimes \gamma_{2}, \operatorname{\Omega map}_{K}\left(a_{2}, a_{0}\right)\right)$ and $f_{*} \pi_{0} \operatorname{map}_{\mathcal{M}}\left(\gamma_{2} \otimes \gamma_{3}, \Omega_{\mathbf{m a p}_{K}}\left(a_{3}, a_{1}\right)\right)$, where $h$ acts on $\operatorname{map}_{\mathcal{M}}\left(\gamma_{1} \otimes \gamma_{2}, \Omega_{\mathbf{m a p}_{K}}\right.$ $\left(a_{2}, a_{0}\right)$ ) via $\theta^{R}$ (see Definition 2.1, Sect. 2.1 and (2.9)), and similarly for $f$. Compare [61, Sect. 1].

However, for higher order Toda brackets the indeterminacy is too complicated to describe by a single formula, as it depends on various intermediate choices.

\subsection{Massey products in DGAs}

Since $\mathrm{Ch}_{R}$ is a model category, we can consider higher Toda brackets for a differential graded algebra $\mathbf{A}_{*}$, as in Sect. 4.1 (we think of $\mathbf{A}_{*}$ as a chain complex, rather than a cochain complex, but since we allow arbitrary $\mathbb{Z}$-grading, this is no restriction). 
A chain complex $\Lambda$ of length $n+1$ in ho $\mathbf{A}_{*}$ consists of a sequence $\left(\gamma_{i}\right)_{i=1}^{n+1}$ of homology classes in $H_{*} \mathbf{A}_{*}$, with $\gamma_{i} \cdot \gamma_{i+1}=0$ for $i=1, \ldots, n$. If we choose an $n$-th order chain complex (that is, a DGA $\mathbf{A}_{*}$ ) realizing $\Lambda$, as above, we obtain the element given by (4.8) in $\widetilde{\Omega}^{n-1} \mathbf{A}_{*}$. However, because we are working over $\operatorname{Mod}_{R}$ we can define the identification $\widetilde{\Omega}^{n-1} \mathbf{A}_{*} \cong \Omega^{n-1} \mathbf{A}_{*}$ using the Dold-Kan equivalence (essentially, by the homotopy addition theorem—cf. [44]), and thus obtain the value

$$
\sum_{s=0}^{n-1}(-1)^{s} H_{s+1}^{s} \cdot H_{n+1}^{n-1-s} \in A_{j+n-1}
$$

in $\Omega^{n-1} \mathbf{A}_{*}$, which is readily seen to be a $(j+n-1)$-cycle for $j:=\sum_{t=1}^{n} m_{t}$.

By comparing this formula with the classical definition of the higher Massey product (see, e.g., [59, (V.4)]), we find:

Proposition 5.20 The higher Toda brackets in a differential graded algebra $\mathbf{A}_{*}$ are identical with the usual higher Massey products.

\section{Toda brackets for chain complexes}

We now study Toda brackets in the category $\mathrm{Ch}_{R}^{\geqslant 0}$ of non-negatively graded chain complexes over a hereditary ring $R$, such as $\mathbb{Z}$. It turns out that in this case even ordinary Toda brackets have a finer "homological" structure, which we describe.

\subsection{Chain complexes over hereditary rings}

Since $R$ is hereditary, if $Q_{0}(G)$ is a functorial free cover of an $R$-module $G$, we have a projective presentation

$$
0 \rightarrow Q_{1}(G) \stackrel{\alpha^{G}}{\rightarrow} Q_{0}(G) \stackrel{r}{\rightarrow} G \rightarrow 0
$$

where $Q_{1}(G):=\operatorname{Ker}(r)$.

We then define the $n$-th Moore complex $\mathbf{M}(G, n)_{*}$ for an $R$-module $G$ to be the chain complex with $(M(G, n))_{n+1}:=Q_{1}(G),(M(G, n))_{n}:=Q_{0}(G)$, and 0 otherwise, with $\partial_{n+1}=\alpha^{G}$. This yields a functor $\widehat{C}_{*}: \operatorname{grMod}_{R}^{\geqslant 0} \rightarrow \mathrm{Ch}_{R}^{\geqslant 0}$ with

$$
\widehat{C}_{*}\left(\mathcal{E}_{*}\right):=\bigoplus_{n \geq 0} \mathbf{M}\left(E_{n}, n\right)_{*} .
$$

Recall that $\mathrm{Ch}_{R}^{\geqslant 0}$ has a model structure in which quasi-isomorphisms are the weak equivalences, and a chain complexes is cofibrant if and only if it is projective in each dimension (see [24, Sect. 2.3]). Because $R$ is hereditary, any $\mathbf{A}_{*} \in \mathrm{Ch}_{R}^{\geqslant 0}$ is uniquely determined up to weak equivalence by the graded $R$-module $H_{*} \mathbf{A}_{*}$ (cf. [15, Theorem 3.4]). 
Therefore, if we enrich $\operatorname{grMod}_{R}^{\geqslant 0}$ over $\mathrm{Ch}_{R}$ by setting

$$
\underline{\operatorname{Hom}}\left(\mathcal{E}_{*}, \mathcal{F}_{*}\right):=\underline{\operatorname{Hom}}\left(\widehat{C}_{*}\left(\mathcal{E}_{*}\right), \widehat{C}_{*}\left(\mathcal{F}_{*}\right)\right)
$$

(see Example 2.6), $\widehat{C}_{*}$ becomes an enriched embedding, and in fact:

Lemma 6.1 The functor $\widehat{C}_{*}: \operatorname{grMod}_{R}^{\geqslant 0} \rightarrow \mathrm{Ch}_{R}^{\geqslant 0}$ is a Dwyer-Kan equivalence over $\mathrm{Ch}_{R}$.

Since the right-hand side of (6.1) is a coproduct, we see that $\underline{\operatorname{Hom}}\left(\mathcal{E}_{*}, \mathcal{F}_{*}\right)$ naturally splits as a product

$$
\prod_{n \geq 0}\left(\underline{\operatorname{Hom}}\left(\mathbf{M}\left(E_{n}, n\right)_{*}, \mathbf{M}\left(F_{n}, n\right)_{*}\right) \times \underline{\operatorname{Hom}}\left(\mathbf{M}\left(E_{n}, n\right)_{*}, \mathbf{M}\left(F_{n+1}, n+1\right)_{*}\right)\right) \times P,
$$

where $P$ is a product of similar terms, but with $H_{0} P=0$. Moreover, since

$$
\begin{aligned}
{\left[\mathbf{M}(E, n)_{*}, \mathbf{M}(F, n)_{*}\right] } & \cong \operatorname{Hom}_{R}(E, F) \text { and }\left[\mathbf{M}(E, n)_{*}, \mathbf{M}(F, n+1)_{*}\right] \\
& \cong \operatorname{Ext}_{R}(E, F),
\end{aligned}
$$

we see that (6.2) is an enriched version of the Universal Coefficient Theorem for chain complexes, stating that for chain complexes over a hereditary ring $R$ there is a (split) short exact sequence:

$$
0 \rightarrow \prod_{n>0} \operatorname{Ext}_{R}\left(H_{n-1} \mathbf{A}_{*}, H_{n} \mathbf{B}_{*}\right) \rightarrow\left[\mathbf{A}_{*}, \mathbf{B}_{*}\right] \rightarrow \prod_{n \geq 0} \operatorname{Hom}_{R}\left(H_{n} \mathbf{A}_{*}, H_{n} \mathbf{B}_{*}\right) \rightarrow 0
$$

(cf. [16, Corollary 10.13]). Note that in our version for $\operatorname{grMod}_{R}^{\geqslant 0}$, the splitting is natural!

Notation 6.1 From (6.2) we see that there are two kinds of indecomposable maps of chain complexes (and their nullhomotopies) (see (5.7)):

(a) 'Hom-type' maps $H(f): \mathbf{M}(E, n)_{*} \rightarrow \mathbf{M}(F, n)_{*}$, determined by

$$
f_{n}^{00}: Q_{0}(E) \rightarrow Q_{0}(F) \text { and } f_{n}^{11}: Q_{1}(E) \rightarrow Q_{1}(F) .
$$

A nullhomotopy $H(S): H(f) \sim 0$ is given by $S_{n}^{01}: Q_{0}(E) \rightarrow Q_{1}(F)$, the factorization of $f_{n}^{00}$ through $Q_{1}(F) \hookrightarrow Q_{0}(F)$. If it exists, it is unique.

(b) 'Ext-type' maps

$$
E(f): \mathbf{M}(E, n)_{*} \rightarrow \mathbf{M}(F, n+1)_{*},
$$

determined by $f_{n}^{10}: Q_{1}(E) \rightarrow Q_{0}(F)$. A nullhomotopy $E(S): E(f) \sim 0$ is given by $S_{n}^{00}: Q_{0}(E) \rightarrow Q_{0}(F)$ and $S_{n}^{11}: Q_{1}(E) \rightarrow Q_{1}(F)$. 


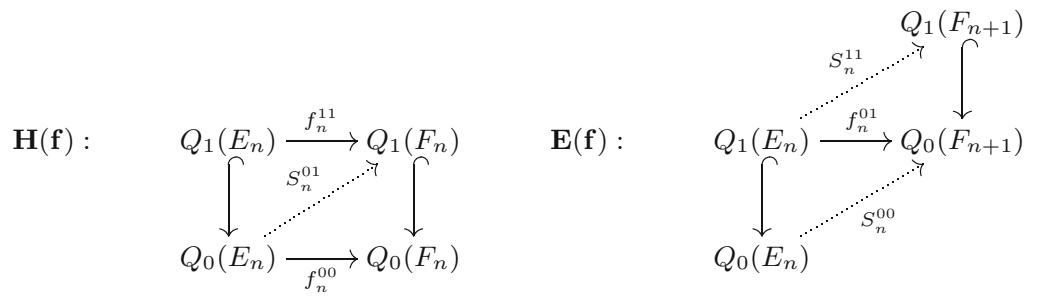

\subsection{Secondary chain complexes in $\operatorname{grMod}_{R}^{\geqslant 0}$}

In light of the above discussion, we see that any secondary chain complex

$$
\widehat{C}_{*}\left(\mathcal{E}_{*}\right) \stackrel{f}{\rightarrow} \widehat{C}_{*}\left(\mathcal{F}_{*}\right) \stackrel{g}{\rightarrow} \widehat{C}_{*}\left(\mathcal{G}_{*}\right) \stackrel{h}{\rightarrow} \widehat{C}_{*}\left(\mathcal{H}_{*}\right)
$$

in the $\mathrm{Ch}_{R}$-enriched category grMod $\geqslant 0$ is a direct sum of secondary chain complexes of one of the following four elementary forms:

$$
\begin{gathered}
\mathbf{M}\left(E_{n}, n\right)_{*} \stackrel{H(f) \top E(f)}{\longrightarrow} \mathbf{M}\left(F_{n}, n\right)_{*} \oplus \mathbf{M}\left(F_{n+1}, n+1\right)_{*} \\
\stackrel{E(g) \perp H(g)}{\longrightarrow} \mathbf{M}\left(G_{n+1}, n+1\right)_{*} \stackrel{H(h)}{\longrightarrow} \mathbf{M}\left(H_{n+1}, n+1\right)_{*}, \\
\mathbf{M}\left(E_{n}, n\right)_{*} \stackrel{H(f) \top E(f)}{\longrightarrow} \mathbf{M}\left(F_{n}, n\right)_{*} \oplus \mathbf{M}\left(F_{n+1}, n+1\right)_{*} \\
\stackrel{E(g) \perp H(g)}{\longrightarrow} \mathbf{M}\left(G_{n+1}, n+1\right)_{*} \stackrel{E(h)}{\longrightarrow} \mathbf{M}\left(H_{n+2}, n+2\right)_{*},
\end{gathered}
$$

$$
\begin{aligned}
& \mathbf{M}\left(E_{n}, n\right)_{*} \stackrel{H(f)}{\longrightarrow} \mathbf{M}\left(F_{n}, n\right)_{*} \\
& \quad \stackrel{H(g) \top E(g)}{\longrightarrow} \mathbf{M}\left(G_{n}, n\right)_{*} \oplus \mathbf{M}\left(G_{n+1}, n+1\right)_{*} \stackrel{E(h) \perp H(h)}{\longrightarrow} \mathbf{M}\left(H_{n+1}, n+1\right)_{*}
\end{aligned}
$$

$$
\begin{aligned}
& \mathbf{M}\left(E_{n}, n\right)_{*} \stackrel{E(f)}{\longrightarrow} \mathbf{M}\left(F_{n+1}, n+1\right)_{*} \stackrel{H(g) \top E(g)}{\longrightarrow} \mathbf{M}\left(G_{n+1}, n+1\right)_{*} \\
& \quad \mathbf{M}\left(G_{n+2}, n+2\right)_{*} \stackrel{E(h) \perp H(h)}{\longrightarrow} \mathbf{M}\left(H_{n+2}, n+2\right)_{*}
\end{aligned}
$$

Two additional hypothetical forms, namely:

(i) $\mathbf{M}\left(E_{n}, n\right)_{*} \stackrel{H(f)}{\longrightarrow} \mathbf{M}\left(F_{n}, n\right)_{*} \stackrel{H(g)}{\longrightarrow} \mathbf{M}\left(G_{n}, n\right)_{*} \stackrel{H(h)}{\longrightarrow} \mathbf{M}\left(H_{n}, n\right)_{*}$

(ii) $\mathbf{M}\left(E_{n}, n\right)_{*} \stackrel{E(f)}{\longrightarrow} \quad \mathbf{M}\left(F_{n+1}, n+1\right)_{*} \stackrel{E(g)}{\longrightarrow} \quad \mathbf{M}\left(G_{n+2}, n+2\right)_{*} \quad \stackrel{E(h)}{\longrightarrow}$ $\mathbf{M}\left(H_{n+3}, n+3\right)_{*}$

in fact are irrelevant to Toda brackets, for dimensional reasons.

Moreover, the four elementary secondary chain complexes may or may not split further into one of the following six atomic forms: 
(a) $\mathbf{M}\left(E_{n}, n\right)_{*} \stackrel{H(f)}{\longrightarrow} \mathbf{M}\left(F_{n}, n\right)_{*} \stackrel{H(g)}{\longrightarrow} \mathbf{M}\left(G_{n}, n\right)_{*} \stackrel{E(h)}{\longrightarrow} \mathbf{M}\left(H_{n+1}, n+1\right)_{*}$ and two similar cases with a single $E$-term;

(b) $\mathbf{M}\left(E_{n}, n\right)_{*} \stackrel{H(f)}{\longrightarrow} \mathbf{M}\left(F_{n}, n\right)_{*} \stackrel{E(g)}{\longrightarrow} \mathbf{M}\left(G_{n+1}, n+1\right)_{*} \stackrel{E(h)}{\longrightarrow} \mathbf{M}\left(H_{n+2}, n+2\right)_{*}$ and two similar cases with a single $H$-term.

\subsection{Secondary Toda brackets in $\operatorname{grMod}_{R}^{\geqslant 0}$}

By Definition 5.17, a secondary Toda bracket in the $\mathrm{Ch}_{R}$-enriched category $\operatorname{grMod} \geqslant 0$ is associated to a homotopy chain complex $\Lambda$ of length 3 in ho $\operatorname{grMod}_{R}^{\geqslant 0}$ as in (5.14). This means that we replace the actual chain maps in each of the twelve examples of Sect. 6.2 by their homotopy classes: that is, elements in $\operatorname{Hom}_{R}(E, F) \operatorname{or}_{\operatorname{Ext}_{R}}(E, F)$, respectively.

The compositions $\operatorname{Hom}(E, F) \otimes \operatorname{Ext}(F, G) \rightarrow \operatorname{Ext}(E, G), \operatorname{Ext}(E, F) \otimes \operatorname{Hom}(F, G)$ $\rightarrow \operatorname{Ext}(E, G)$ simply define the functoriality of $\operatorname{Ext}$, while $\operatorname{Ext}(E, F) \otimes \operatorname{Ext}(F, G) \rightarrow$ $\operatorname{Ext}(E, G)$ vanishes for dimension reasons. Nevertheless, the associated Toda bracket may be non-trivial.

Note that in this case, as in the original construction of Toda in [61] (see also [56]), the subset $\langle\langle\Lambda\rangle\rangle$ of $\left[\Sigma \mathcal{E}_{*}, \mathcal{H}_{*}\right]$ is actually a double coset of the group

$$
(\Sigma f)^{\sharp}\left[\Sigma \mathcal{F}_{*}, \mathcal{H}_{*}\right]+h_{\sharp}\left[\Sigma \mathcal{E}_{*}, \mathcal{G}_{*}\right],
$$

so we can think of $\langle\langle\Lambda\rangle\rangle$, which we usually denote simply by $\langle h, g, f\rangle$, as taking value in the quotient abelian group

$$
\langle h, g, f\rangle \in(\Sigma f)^{\sharp}\left[\Sigma \mathcal{F}_{*}, \mathcal{H}_{*}\right] \backslash\left[\Sigma \mathcal{E}_{*}, \mathcal{H}_{*}\right] / h_{\sharp}\left[\Sigma \mathcal{E}_{*}, \mathcal{G}_{*}\right] .
$$

Thus the elementary examples of Sect. 6.2 may be interpreted as secondary operations in Ext $_{R}$, defined under certain vanishing assumptions, and with an explicit indeterminacy (which may be less than that indicated in (6.9) in any specific case).

For example, in (6.6) (case (e) above), the operation is defined for elements in the pullback of

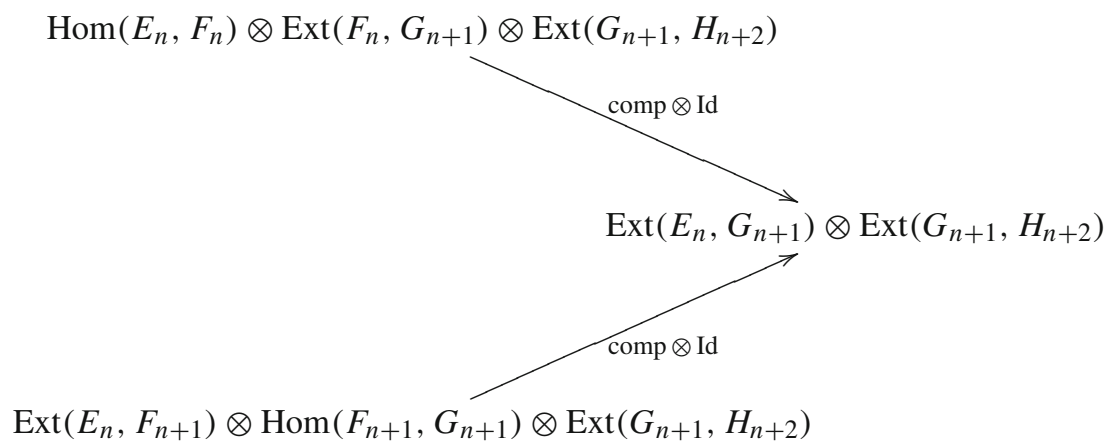


and takes value in the quotient $\operatorname{group} \operatorname{Ext}\left(E_{n}, H_{n+1}\right) / h_{\sharp} \operatorname{Hom}\left(E_{n}, G_{n}\right)$, where $h_{\sharp} \operatorname{Hom}\left(E_{n}, G_{n}\right)$ refers to the image of the given element $h \in \operatorname{Ext}\left(G_{n}, H_{n+1}\right)$ under precomposition with all elements of $\operatorname{Hom}\left(E_{n}, G_{n}\right)$.

It turns out that cases (a) and (d) are trivial for dimension reasons, but we shall now provide examples of non-triviality for four of the remaining cases.

Example 6.2 Consider the homotopy chain complex $\Lambda$ in ho $\operatorname{grMod}_{R}^{\geqslant 0}$ given by $E_{0}=$ $\mathbb{Z} / 2, F_{0}=\mathbb{Z} / 4, G_{0}=\mathbb{Z} / 2$, and $H_{1}=\mathbb{Z} / 2$, with the corresponding maps

$$
\begin{aligned}
& f=2 \in \mathbb{Z} / 2=\operatorname{Hom}\left(E_{0}, F_{0}\right)=\operatorname{Hom}(\mathbb{Z} / 2, \mathbb{Z} / 4) \\
& g=1 \in \mathbb{Z} / 2=\operatorname{Hom}\left(F_{0}, G_{0}\right)=\operatorname{Hom}(\mathbb{Z} / 4, \mathbb{Z} / 2) \\
& h=2 \in \mathbb{Z} / 2=\operatorname{Ext}\left(F_{0}, H_{1}\right)=\operatorname{Ext}(\mathbb{Z} / 4, \mathbb{Z} / 2) .
\end{aligned}
$$

By Lemma 5.18, we may choose any cofibrant chain complexes in $\mathrm{Ch}_{\mathbb{Z}}$ to realize $\Lambda$, not necessarily the functorial versions $\widehat{C}_{*}\left(\mathcal{E}_{*}\right)$, and so on. In our case we shall use the following minimal secondary chain complex:

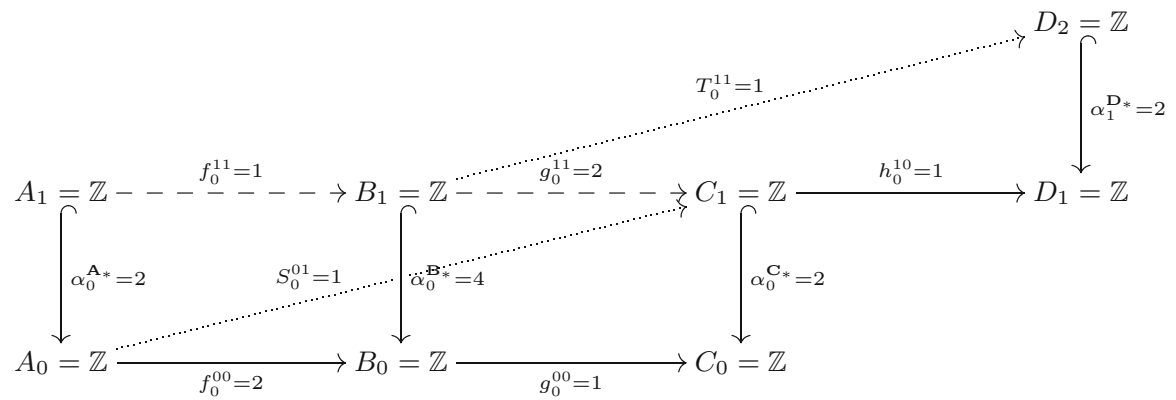

The Toda bracket is given by:

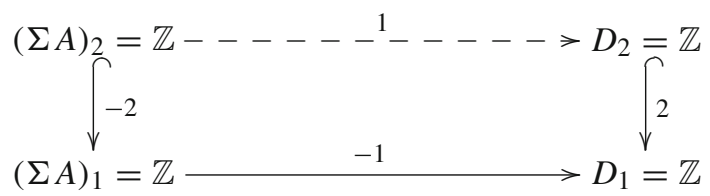

The indeterminacy is given by

$$
\begin{aligned}
(\Sigma f)^{\sharp}\left[\Sigma \mathcal{F}_{*}, \mathcal{H}_{*}\right]+h_{\sharp}\left[\Sigma \mathcal{E}_{*}, \mathcal{G}_{*}\right] & =\Sigma f^{\sharp} \operatorname{Hom}\left(F_{0}, H_{1}\right)+h_{\sharp} \operatorname{Hom}\left(E_{0}, G_{1}\right) \\
& =2 \cdot(\mathbb{Z} / 2)+0=0 .
\end{aligned}
$$

Hence the Toda bracket $\langle h, g, f\rangle$ does not vanish.

Example 6.3 Consider the homotopy chain complex in ho grMod $\geqslant_{R}^{\geqslant 0}$ given by $E_{0}=$ $\mathbb{Z} / 2, F_{1}=\mathbb{Z} / 4, G_{1}=\mathbb{Z} / 4$, and $H_{2}=\mathbb{Z}$, with the corresponding maps $f=1 \in$ $\mathbb{Z} / 2=\operatorname{Ext}(\mathbb{Z} / 2, \mathbb{Z} / 4), g=2 \in \mathbb{Z} / 4=\operatorname{Hom}(\mathbb{Z} / 4, \mathbb{Z} / 4)$, and $h=2 \in \mathbb{Z} / 4=$ $\operatorname{Ext}(\mathbb{Z} / 4, \mathbb{Z})$.

We choose the following associated secondary chain complex: 


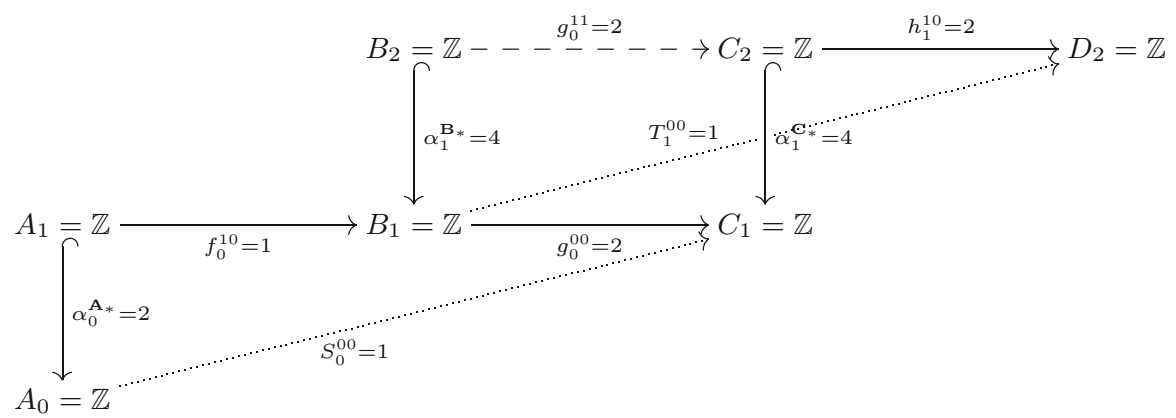

The Toda bracket is represented as follows:

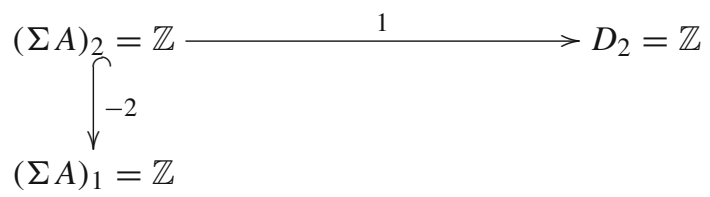

which is a generator of $\operatorname{Ext}(\mathbb{Z} / 2, \mathbb{Z})=\mathbb{Z} / 2$. The indeterminacy is

$$
\begin{aligned}
(\Sigma f)^{\sharp}\left[\Sigma \mathcal{F}_{*}, \mathcal{H}_{*}\right]+h_{\sharp}\left[\Sigma \mathcal{E}_{*}, \mathcal{G}_{*}\right] & =\Sigma f^{\sharp} \operatorname{Hom}\left(F_{0}, H_{1}\right)+h_{\sharp} \operatorname{Hom}\left(E_{0}, G_{1}\right) \\
& =1 \cdot 0+2 \cdot(\mathbb{Z} / 2)=0 .
\end{aligned}
$$

Hence the Toda bracket $\langle h, g, f\rangle$ does not vanish.

Example 6.4 Consider the homotopy chain complex in ho grMod $\geqslant_{R}^{\geqslant 0}$ given by $E_{0}=$ $\mathbb{Z} / 8, F_{1}=\mathbb{Z} / 4, G_{1}=\mathbb{Z} / 4$, and $H_{2}=\mathbb{Z}$, with the corresponding maps $f=1 \in$ $\mathbb{Z} / 4=\operatorname{Hom}(\mathbb{Z} / 8, \mathbb{Z} / 4), g=2 \in \mathbb{Z} / 4=\operatorname{Ext}(\mathbb{Z} / 4, \mathbb{Z} / 4)$, and $h=1 \in \mathbb{Z} / 4=$ $\operatorname{Ext}(\mathbb{Z} / 4, \mathbb{Z})$.

We may choose the following associated secondary chain complex:

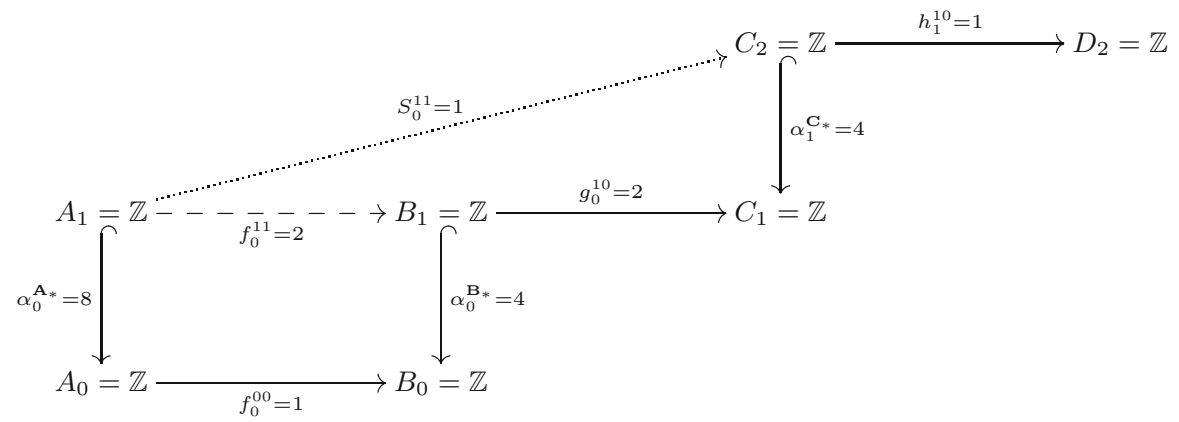

The Toda bracket is given by:

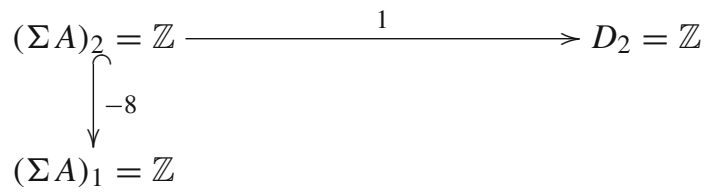


which is a generator of $\operatorname{Ext}(\mathbb{Z} / 8, \mathbb{Z})=\mathbb{Z} / 8$. The indeterminacy is

$$
(\Sigma f)^{\sharp}\left[\Sigma \mathcal{F}_{*}, \mathcal{H}_{*}\right]+h_{\sharp}\left[\Sigma \mathcal{E}_{*}, \mathcal{G}_{*}\right]=f^{\sharp} \operatorname{Ext}\left(F_{0}, H_{2}\right)+h_{\sharp} \operatorname{Hom}\left(E_{0}, G_{1}\right) .
$$

A generator of $f^{\sharp} \operatorname{Ext}\left(F_{0}, H_{2}\right)=1 \cdot \operatorname{Ext}(\mathbb{Z} / 4, \mathbb{Z})=\mathbb{Z} / 4$ in $\operatorname{Ext}\left(E_{0}, H_{2}\right)$ is given by

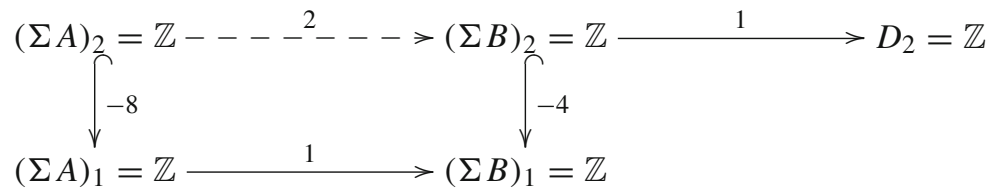

while a generator of $h_{\sharp} \operatorname{Hom}\left(E_{0}, G_{1}\right)=1 \cdot \operatorname{Hom}(\mathbb{Z} / 8, \mathbb{Z} / 4)=\mathbb{Z} / 4$ in $\operatorname{Ext}\left(E_{0}, H_{2}\right)$ is given by

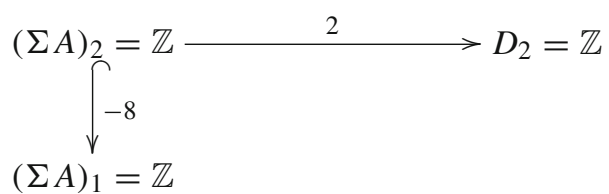

so the total indeterminacy is the subgroup $\mathbb{Z} / 4 \subseteq \mathbb{Z} / 8=\operatorname{Ext}(\mathbb{Z} / 8, \mathbb{Z})=\operatorname{Ext}\left(E_{0}, H_{2}\right)$. Since the Toda bracket $\langle h, g, f\rangle$ is represented by a generator of this $\mathbb{Z} / 8$, it does not vanish.

Example 6.5 Consider the homotopy chain complex in ho grMod $\geqslant_{R}^{\geqslant 0}$ given by $E_{0}=$ $\mathbb{Z} / 16, F_{0}=\mathbb{Z} / 8, F_{1}=\mathbb{Z} / 16, G_{1}=\mathbb{Z} / 16$, and $H_{1}=\mathbb{Z} / 16$, with the corresponding maps $f=1 \in \mathbb{Z} / 8=\operatorname{Hom}\left(E_{0}, F_{0}\right), f^{\prime} \in \mathbb{Z} / 16=\operatorname{Ext}\left(E_{0}, F_{1}\right), g=4 \in \mathbb{Z} / 8=$ $\operatorname{Ext}\left(F_{0}, G_{1}\right), g^{\prime} \in \mathbb{Z} / 16=\operatorname{Hom}\left(F_{1}, G_{1}\right)$ and $h=2 \in \mathbb{Z} / 16=\operatorname{Hom}\left(G_{1}, H_{1}\right)$.

We may choose the following associated secondary chain complex:

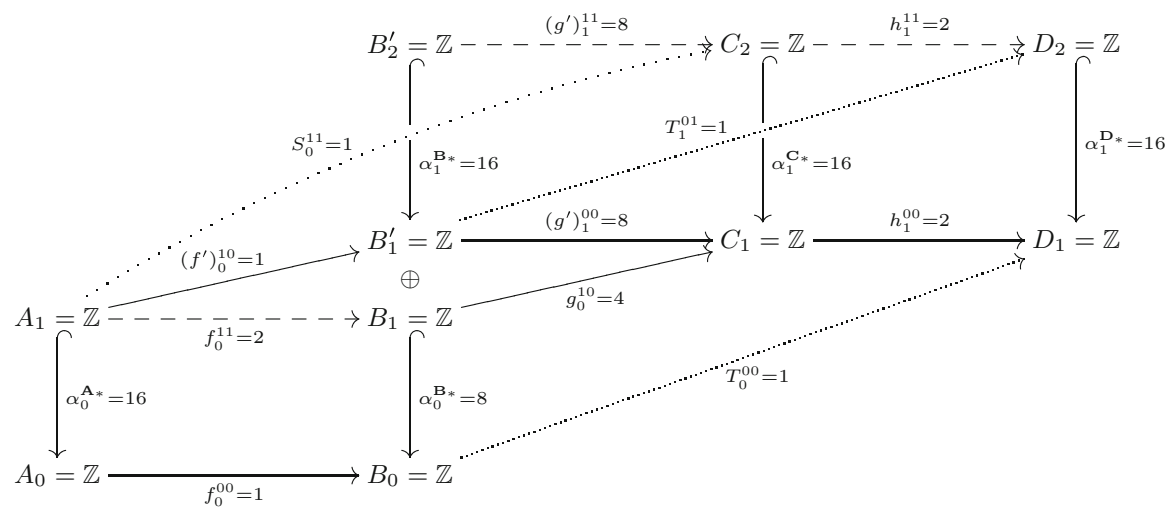


The Toda bracket is given by:

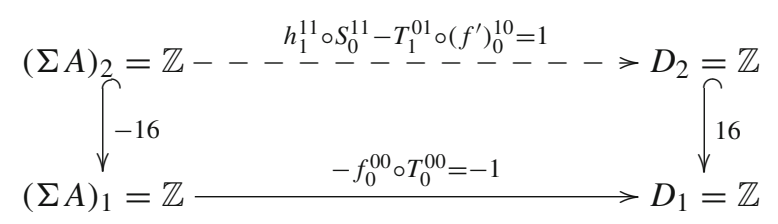

which is a generator of $\operatorname{Hom}(\mathbb{Z} / 16, \mathbb{Z} / 16)=\mathbb{Z} / 16$. The indeterminacy is

$$
(\Sigma f)^{\sharp}\left[\Sigma \mathcal{F}_{*}, \mathcal{H}_{*}\right]+h_{\sharp}\left[\Sigma \mathcal{E}_{*}, \mathcal{G}_{*}\right]=f^{\sharp} \operatorname{Hom}\left(F_{0}, H_{1}\right)+h_{\sharp} \operatorname{Hom}\left(E_{0}, G_{1}\right) .
$$

A generator of $f^{\sharp} \operatorname{Hom}\left(F_{0}, H_{1}\right)=1 \cdot \operatorname{Hom}(\mathbb{Z} / 8, \mathbb{Z} / 16)=\mathbb{Z} / 8$ in $\operatorname{Hom}\left(E_{0}, H_{1}\right)$ is given by

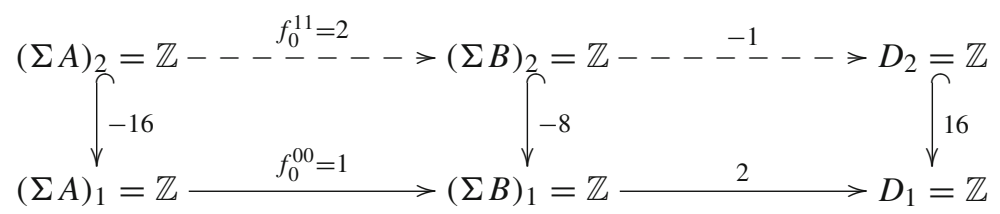

while a generator of $h_{\sharp} \operatorname{Hom}\left(E_{0}, G_{1}\right)=2 \cdot \operatorname{Hom}(\mathbb{Z} / 16, \mathbb{Z} / 16)=\mathbb{Z} / 8$ in $\operatorname{Hom}\left(E_{0}, H_{1}\right)$ is given by

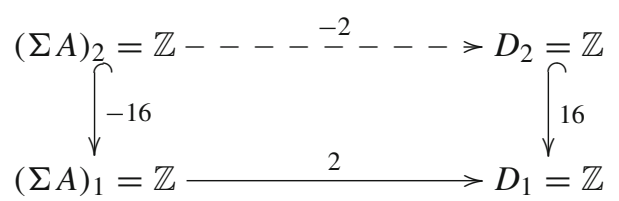

so the Toda bracket $\langle h, g, f\rangle$ does not vanish.

Remark 6.6 See [7, Sect. 6.12] for a calculation relating Toda brackets in topology with a certain operation in homological algebra.

Acknowledgements We wish to thank the referee for many useful comments, and Stefan Schwede for a helpful pointer on symmetric spectra. The research of the second author was supported by Israel Science Foundation Grant No. 47377, and he also wishes to thank the Max Planck Institute for Mathematics for their hospitality during several visits. The third author was supported by a Vatat fellowship during the period of this research.

\section{References}

1. Adams, J.F.: On the structure and applications of the Steenrod algebra. Commun. Math. Helv. 32, 180-214 (1958)

2. Adams, J.F.: On the non-existence of elements of Hopf invariant one. Ann. Math. 72(1), 20-104 (1960)

3. Alexander, J.C.: Cobordism Massey products. Trans. AMS 166, 197-214 (1972)

4. Babenko, I.K., Taŭmanov, I.A.: Massey products in symplectic manifolds. Mat. Sb. 191, 3-44 (2000) 
5. Barratt, M.G., Jones, J.D.S., Mahowald, M.E.: Relations amongst Toda brackets and the Kervaire invariant in dimension 64. J. Lond. Math. Soc. 30, 533-550 (1984)

6. Baues, H.J.: Obstruction Theory on Homotopy Classification of Maps. Lec. Notes Math., vol. 628. Springer, Berlin, New York (1977)

7. Baues, H.-J.: Homotopy type and Homology. Oxford Mathematical Monographs, New York (1996)

8. Baues, H.J.: The Algebra of secondary cohomology operations, Progress in Math., vol. 247. Birkhäuser, USA (2006)

9. Baues, H.-J., Blanc, D.: Higher order derived functors and the Adams spectral sequence. J. Pure Appl. Alg. 219, 199-239 (2015)

10. Benson, D., Krause, H., Schwede, S.: Introduction to realizability of modules over Tate cohomology. In: Representations of algebras and related topics. AMS, Providence, RI (2005)

11. Berger, C., Moerdijk, I.: On the homotopy theory of enriched categories. Q. J. Math. 64, 805-846 (2013)

12. Blanc, D., Johnson, M.W., Turner, J.M.: Higher homotopy operations and cohomology. J. K Theory 5, 167-200 (2010)

13. Blanc, D., Johnson, M.W., Turner, J.M.: Higher homotopy operations and André-Quillen cohomology. Adv. Math. 230, 777-817 (2012)

14. Boardman, J.M., Vogt, R.M.: Homotopy Invariant Algebraic Structures on Topological Spaces. Lec. Notes Math., vol. 347. Springer, Berlin, New York (1973)

15. Dold, A.: Homology of symmetric products and other functors of complexes. Ann. Math. 68(2), 54-80 (1958)

16. Dold, A.: Lectures on algebraic topology. Springer, Berlin (1995)

17. Elmendorf, A.D., Křiž, I., Mandell, M.A., May, J.P.: Rings, modules, and algebras in stable homotopy theory. AMS, Providence, RI (1997)

18. Fuchs, D., Weldon, L.L.: Massey brackets and deformations. J. Pure Appl. Alg. 156, 215-229 (2001)

19. Grant, M.: "Topological complexity of motion planning and Massey products". In: Algebraic topologyold and new, pp. 193-203. Polish Acad. Sci. Inst. Math., Warsaw (2009)

20. Hardie, K.A., Kamps, K.H., Marcum, H.J.: A categorical approach to matrix Toda brackets. Trans. AMS 347, 4625-4649 (1995)

21. Heller, A.: Stable homotopy categories. Bull. AMS 74, 28-63 (1968)

22. Hirschhorn, P.S.: Model Categories and their Localizations. Math. Surveys \& Monographs, vol. 99. AMS, Providence, RI (2002)

23. Hopkins, M.J., Wickelgren, K.: Splitting Varieties for Triple Massey Products. J. Pure Appl. Alg. 219, 1304-1319 (2015)

24. Hovey, M.A.: Model Categories. Math. Surveys \& Monographs, vol. 63. AMS, Providence, RI (1998)

25. Hovey, M.A., Shipley, B.E., Smith, J.H.: Symmetric spectra. J. AMS 13, 149-208 (2000)

26. Kim, M.: Massey products for elliptic curves of rank 1. J. AMS 23, 725-747 (2010)

27. Klaus, S.: Towers and Pyramids, I. Fund. Math 13(5), 663-683 (2001)

28. Kraines, D.P.: Massey higher products. Trans. AMS 124, 431-449 (1966)

29. Kristensen, L.: On secondary cohomology operations. Math. Scand. 12, 57-82 (1963)

30. Kristensen, L., Madsen, I.H.: On evaluation of higher order cohomology operations. Math. Scand. 20, 114-130 (1967)

31. Laures, G.: Toda brackets and congruences of modular forms. Algebraic Geom. Top. 11, 1893-1914 (2011)

32. Laurence,P., Stredulinsky, E.: A lower bound for the energy of magnetic fields supported in linked tori. C. R. Acad. Sci. Paris I Math. 331, 201-206 (2000)

33. Lurie, J.: Higher Topos Theory. Princeton University Press, Princeton (2009)

34. Mac Lane, S.: Categories for the Working Mathematician. Grad. Texts in Math. vol. 5. Springer, Berlin, New York (1971)

35. Mahowald, M.E., Peterson, F.P.: Secondary operations on the Thom class. Topology 2, 367-377 (1964)

36. Mandell, M.A., May, J.P., Schwede, S., Shipley, B.: Model categories of diagram spectra. Proc. Lond. Math. Soc. 82(3), 441-512 (2001)

37. Marcum, H.J., Oda, N.: Long box bracket operations in homotopy theory. Appl. Categorical Struct. 19, 137-173 (2011)

38. Margolis, H.R.: Spectra and the Steenrod Algebra: Modules over the Steenrod Algebra and the Stable Homotopy Category. North-Holland, Amsterdam, New York (1983)

39. Massey, W.S.: A new cohomology invariant of topological spaces. Bull. AMS 57, 74 (1951) 
40. Mather, M.: Pull-backs in homotopy theory. Can. J. Math. 28, 225-263 (1976)

41. Maunder, C.R.F.: Cohomology operations of the $N$-th kind. Proc. Lond. Math. Soc. 13(2), 125-154 (1963)

42. Mizuno, T.: On a generalization of Massey products. J. Math. Kyoto Univ. 48, 639-659 (2008)

43. Mori, M.: On higher Toda brackets. Bull. Coll. Sci. Univ. Ryukyus 35, 1-4 (1983)

44. Munkres, J.R.: The special homotopy addition theorem. Mich. Math. J, 2, 127-134 (1953/1954)

45. Neisendorfer, J.A.: Primary homotopy theory. Mem. AMS, vol. 25. AMS, Providence, RI (1980)

46. Ōguchi, K.: A generalization of secondary composition and applications. J. Fac. Sci. Univ. Tokyo Sect. I(10), 29-79 (1963)

47. Peterson, F.P., Stein, N.: Secondary cohomology operations: two formulas. Am. J. Math. 81, 231-305 (1959)

48. Porter, G.J.: Higher order Whitehead products. Topology 3, 123-165 (1965)

49. Porter, G.J.: Higher products. Trans. AMS 148, 315-345 (1970)

50. Quillen, D.G.: Homotopical Algebra, Lec. Notes Math., vol. 20. Springer, Berlin, New York (1963)

51. Retakh, V.S.: Lie-Massey brackets and $n$-homotopically multiplicative maps of differential graded Lie algebras. J. Pure Appl. Alg. 89(1-2), 217-229 (1993)

52. Rizzi, C.: Infinitesimal invariant and Massey products. Man. Math. 127, 235-248 (2008)

53. Sagave, S.: Universal Toda brackets of ring spectra. Trans. AMS 360, 2767-2808 (2008)

54. Schwede, S., Shipley, B.: Equivalences of monoidal model categories. Algebraic Geom. Topol. 3, 287-334 (2003)

55. Sharifi, R.T.: Massey products and ideal class groups. J. Reine Angew. Math. 603, 1-33 (2007)

56. Spanier, E.H.: Secondary operations on mappings and cohomology. Ann. Math. 75(2), 260-282 (1962)

57. Spanier, E.H.: Higher order operations. Trans. AMS 109, 509-539 (1963)

58. Steenrod, N.E.: A convenient category of topological spaces. Mich. Math. J 14, 133-152 (1967)

59. Tanré, D.: Homotopie Rationelle: Modèles de Chen, Quillen, Sullivan. Lec. Notes Math. vol. 1025. Springer, Berlin, New York (1983)

60. Toda, H.: Generalized Whitehead products and homotopy groups of spheres. J. Inst. Polytech. Osaka City Univ. Ser. A Math. 3, 43-82 (1952)

61. Toda, H: Composition methods in the homotopy groups of spheres. Adv. in Math. Study, vol. 49. Princeton University Press, Princeton (1962)

62. Vogt, R.M.: Convenient categories of topological spaces for homotopy theory. Arch. Math. (Basel) 22, 545-555 (1971)

63. Walker, G.: Long Toda brackets. In: Proceedings of the Advanced Study Institute on Algebraic Topology, vol. III (Aarhus, 1970), pp. 612-631. Aarhus (1970)

64. Weibel, C.A.: An Introduction to Homological Algebra. Cambridge Uninveristy Press, Cambridge (1994) 